\title{
Assessments of F16 Special Sensor Microwave Imager and Sounder Antenna Temperatures at Lower Atmospheric Sounding Channels
}

\author{
Banghua Yan and Fuzhong Weng \\ Perot System Inc., Joint Center for Satellite Data Assimilation, Center for Satellite Applications and Research (STAR), \\ NOAA Satellites and Information, National Environmental Satellite, Data, and Information Service, 5200 Auth Road, Room 703, \\ Camp Springs, MD 20746, USA \\ Correspondence should be addressed to Banghua Yan, banghua.yan@noaa.gov
}

Received 15 August 2008; Accepted 22 December 2008

Recommended by Zhanqing Li

The main reflector of the Special Sensor Microwave Imager/Sounder (SSMIS) aboard the Defense Meteorological Satellite Program (DMSP) F-16 satellite emits variable radiation, and the SSMIS warm calibration load is intruded by direct and indirect solar radiation. These contamination sources produce antenna brightness temperature anomalies of around $2 \mathrm{~K}$ at SSMIS sounding channels which are obviously inappropriate for assimilation into numerical weather prediction models and remote sensing retrievals of atmospheric and surface parameters. In this study, antenna brightness temperature anomalies at several lower atmospheric sounding (LAS) channels are assessed, and the algorithm is developed for corrections of these antenna temperature anomalies. When compared against radiative transfer model simulations and simultaneous observations from AMSU-A aboard NOAA-16, the SSMIS antenna temperatures at 52.8, 53.6, 54.4, 55.5, 57.3, and $59.4 \mathrm{GHz}$ after the anomaly correction exhibit small residual errors $(<0.5 \mathrm{~K})$. After such SSMIS antenna temperatures are applied to the National Center for Environmental Prediction Numerical Weather Prediction (NWP) model, more satellite data is used and the analysis field of the geopotential height is significantly improved throughout troposphere and lower stratosphere. Therefore, the SSMIS antenna temperatures after the anomaly correction have demonstrated their potentials in NWP models.

Copyright () 2009 B. Yan and F. Weng. This is an open access article distributed under the Creative Commons Attribution License, which permits unrestricted use, distribution, and reproduction in any medium, provided the original work is properly cited.

\section{Introduction}

On October 18, 2003, the F16 satellite was successfully launched and carried the first Special Sensor Microwave Imager/Sounder (SSMIS). The SSMIS instrument has twenty four channels to measure the Earth's radiation at frequencies between 19 and $183 \mathrm{GHz}$ [1]. It is the first operational microwave satellite radiometer for profiling temperature and humidity using a conical scanning mode so that the viewing area and slant path remain nearly constant as it scans the earth. The SSMIS observations also allow simultaneous retrievals of surface and atmospheric parameters with a uniform spatial resolution across the scan swath. However, the accuracy of SSMIS-derived surface and atmospheric products is critically dependent on the accuracy of the brightness temperature measurements.
National Oceanic and Atmospheric Administration (NOAA) receives the F16 SSMIS data in both antenna brightness temperature data record (TDR) and sensor brightness temperature data record (SDR) formats through a data sharing agreement with the Defense Meteorological Satellite Program (DMSP). NOAA then distributes the data to the international user community through the National Environmental Satellites, Data, and Information Service (NESDIS). Here, TDR data contains observation time, geolocation, earth-viewing antenna temperatures, cold counts, warm counts, and so forth, whereas SDR data contains the observation time, geolocation, earth-viewing brightness temperatures [2]. Soon after launch of the SSMIS, some anomalies were discovered in the TDR data for sounding channels primarily due to (a) thermal emission by the main reflector and (b) unaccounted solar radiation impinging 
TABLE 1: Channel characteristics of SSMIS sensor [1]. ((1) Sampling refers to along scan direction based on $833 \mathrm{~km}$ spacecraft altitude. (2) NEDT for instrument temperature $0^{\circ} \mathrm{C}$ and calibration target $260 \mathrm{~K}$ with integration times of $8.4 \mathrm{msec}$ for Channels $12-16$; $12.6 \mathrm{msec}$ for Channels 1-7, 24; and $25.2 \mathrm{msec}$ for Channels 19-23 and $4.2 \mathrm{msec}$ for Channels 8-11, 17-18. (3) Number of subbands is indicated by ( $n$ ) next to individual 3-db width. (4) RCP denotes right-hand circular polarization.)

\begin{tabular}{|c|c|c|c|c|c|c|}
\hline Channel & Center freq. (GHz) & 3-db width $(\mathrm{MHz})$ & Freq. stab. (MHz) & Pol. & $\operatorname{NEDT}(\mathrm{K})$ & Sampling interval $(\mathrm{km})$ \\
\hline 1 & 50.3 & 380 & 10 & $\mathrm{~V}$ & 0.34 & 37.5 \\
\hline 2 & 52.8 & 389 & 10 & $\mathrm{~V}$ & 0.32 & 37.5 \\
\hline 3 & 53.596 & 380 & 10 & $\mathrm{~V}$ & 0.33 & 37.5 \\
\hline 4 & 54.4 & 383 & 10 & $\mathrm{~V}$ & 0.33 & 37.5 \\
\hline 5 & 55.5 & 391 & 10 & $\mathrm{~V}$ & 0.34 & 37.5 \\
\hline 6 & 57.29 & 330 & 10 & $\mathrm{RCP}$ & 0.41 & 37.5 \\
\hline 7 & 59.4 & 239 & 10 & $\mathrm{RCP}$ & 0.40 & 37.5 \\
\hline 8 & 150 & $1642(2)$ & 200 & $\mathrm{H}$ & 0.89 & 12.5 \\
\hline 9 & $183.31 \pm 6.6$ & $1526(2)$ & 200 & $\mathrm{H}$ & 0.97 & 12.5 \\
\hline 10 & $183.31 \pm 3$ & $1019(2)$ & 200 & $\mathrm{H}$ & 0.67 & 12.5 \\
\hline 11 & $183.31 \pm 1$ & $513(2)$ & 200 & $\mathrm{H}$ & 0.81 & 12.5 \\
\hline 12 & 19.35 & 355 & 75 & $\mathrm{H}$ & 0.33 & 25 \\
\hline 13 & 19.35 & 357 & 75 & $\mathrm{~V}$ & 0.31 & 25 \\
\hline 14 & 22.235 & 401 & 75 & $\mathrm{~V}$ & 0.43 & 25 \\
\hline 15 & 37 & 1616 & 75 & $\mathrm{H}$ & 0.25 & 25 \\
\hline 16 & 37 & 1545 & 75 & $\mathrm{~V}$ & 0.20 & 25 \\
\hline 17 & 91.655 & $1418(2)$ & 100 & $\mathrm{~V}$ & 0.33 & 12.5 \\
\hline 18 & 91.655 & $1411(2)$ & 100 & $\mathrm{H}$ & 0.32 & 12.5 \\
\hline 19 & $63.283248 \pm 0.285271$ & $1.35(2)$ & 0.08 & RCP & 2.7 & 75 \\
\hline 20 & $60.792668 \pm 0.357892$ & $1.35(2)$ & 0.08 & RCP & 2.7 & 75 \\
\hline 21 & $60.792668 \pm 0.357892 \pm 0.002$ & $1.3(4)$ & 0.08 & $\mathrm{RCP}$ & 1.9 & 75 \\
\hline 22 & $60.792668 \pm 0.357892 \pm 0.0055$ & $2.6(4)$ & 0.12 & $\mathrm{RCP}$ & 1.3 & 75 \\
\hline 23 & $60.792668 \pm 0.357892 \pm 0.016$ & $7.35(4)$ & 0.34 & RCP & 0.8 & 75 \\
\hline 24 & $60.792668 \pm 0.357892 \pm 0.050$ & $26.5(4)$ & 0.84 & $\mathrm{RCP}$ & 0.9 & 37.5 \\
\hline
\end{tabular}

on the calibration target [3-11]. Similar anomalies are also observed in SSMIS SDR data.

In this study, the anomalies in the SSMIS antenna temperatures at the LAS channels are characterized using the original information provided in the SSMIS TDR. In Section 2, the major characteristics of radiance anomalies at the SSMIS LAS channels are described. Section 3 focuses on the methodology of the algorithm. The SSMIS radiances with anomaly corrections are evaluated in Section 4.

\section{SSMIS Radiance Anomalies at the LAS Channels}

2.1. Brief Description of F16 SSMIS Instrument. The specifications of 24 SSMIS channels are given in Table 1. For reference purposes, the channels are grouped into four subtypes corresponding to the lower atmospheric sounding (LAS) channels (e.g., channels 1-7), the upper atmospheric sounding (UAS) channels (e.g., channels 19-24), the imaging (IMA) channels (i.e., channels 8-11, and 17-18), and the environmental (ENV) channels (e.g., channels 1216), [1]. The SSMIS consists of several major subsystems: antenna/calibration, receiver, signal processing, scan drive, and deployment subsystems. The antenna/calibration subsystem includes the main reflector, an array of feedhorns, a warm calibration load, and a cold calibration reflector. The feedhorn array includes six corrugated feedhorns.

All SSMIS channels are sampled every scan and a sampling period of $4.22 \mathrm{~ms}$ (corresponding to $0.8^{\circ}$ azimuth scan increment) is used for each channel. There are 180 active sample positions where raw radiometric data of earth scenes are acquired from each channel $[2,10]$. These samples except for channels 8-11 and 17-18 are subjected to onboard flight software averaging in the along-scan direction to improve the noise-equivalent difference temperature (NEDT) before down-linking to the ground processing for final TDR calibration. For example, for channels $1-7$ and 24 , three alongscan beams are averaged, resulting in 60 samples per scan; for channels 12-16, two adjacent along-scan beams are averaged, resulting in 90 samples per scan; for channels 19-23, six along-scan beams are averaged, resulting in 30 samples per scan, as summarized in Table 1. For each scan, the feedhorns also pass beneath the stationary warm-load and cold-space reflector calibration targets, where four samples of radiometric counts for each calibration target are taken for all channels and are averaged by the onboard flight software. The temperature of the warm load is provided by three highprecision platinum resistance thermometer (PRT) probes.

Once per rotation of the main reflector, the energy from the Earth's surface and atmosphere incident upon the 


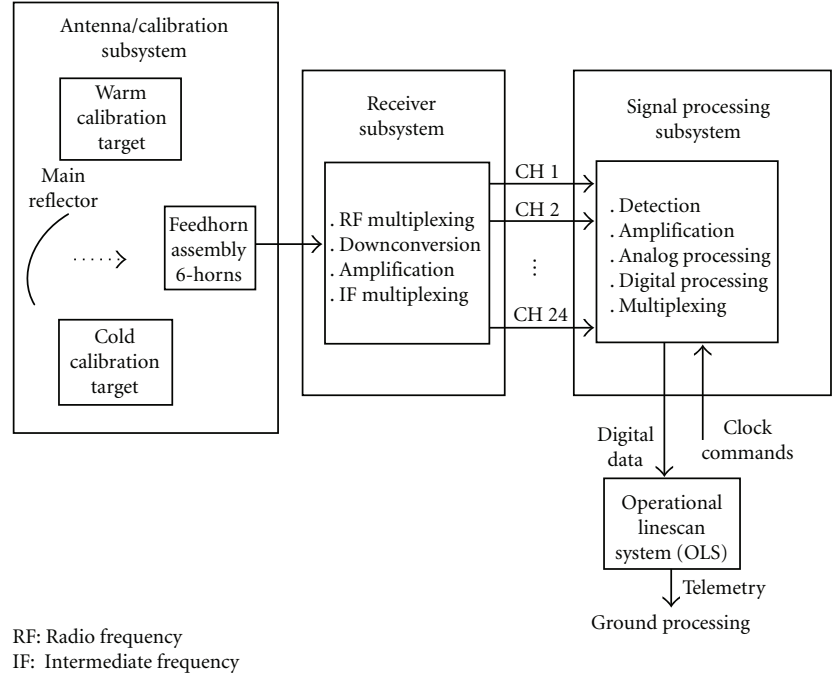

FIGURE 1: SSMIS simplified block diagram [2].

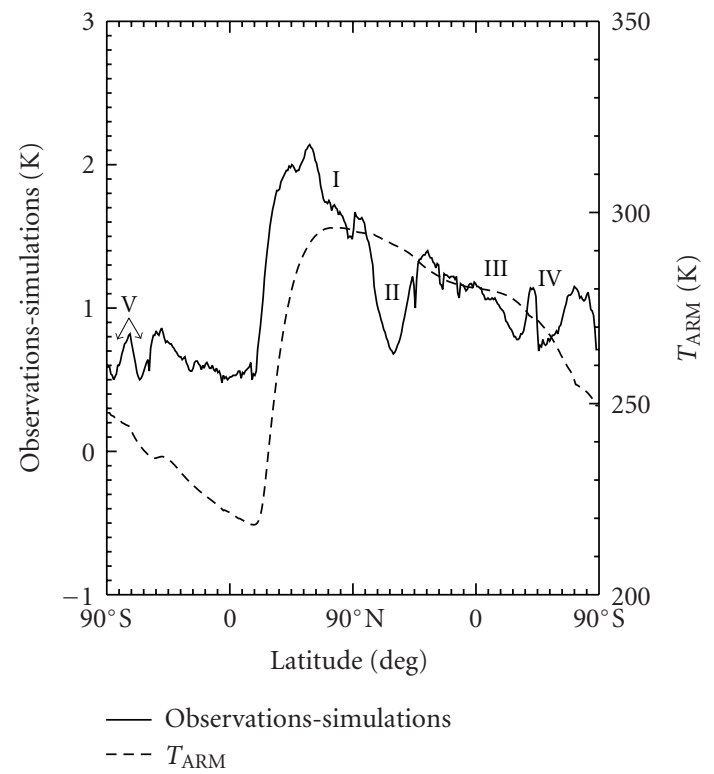

FIgURE 2: The observed arm temperature and the longitudinally antenna temperature differences at $54.4 \mathrm{GHz}$ between the SSMIS TDR measurements and radiative transfer simulations, on March 20,2005 , where the results from $90^{\circ} \mathrm{S}$ to $90^{\circ} \mathrm{N}$ correspond to the ascending node observations and those from $90^{\circ} \mathrm{N}$ to $90^{\circ} \mathrm{S}$ correspond to the descending node observations.

reflector focuses on the feedhorn assembly positioned in the reflector focal plane. The receiver subsystem accepts the energy from the six feedhorns and provided amplification, filtering, and additional frequency multiplexing to output 24 discrete frequency bands to the signal processing subsystem and then to the Operational Linescan System (OLS) under control of the sensor signal processor and the flight software. The data are then transmitted to the SSMIS ground processing by the OLS. Figure 1 displays a block diagram of this process, which is revised from Grumman
[2]. In the SSMIS ground processing, the TDR and SDR are further generated from the Sensor Data Records Processor $[2,10]$. Unfortunately, both main reflector emission and solar heating of the warm calibration target occur in F16 SSMIS such that certain anomalies in the SSMIS antenna temperatures are observed as illustrated next.

2.2. F16 SSMIS Radiance Error Assessment. A powerful approach of monitoring the onorbit satellite instrument performance in satellite cal/val research fields is to compare satellite-observed radiances with radiative transfer model(RTM-) simulated radiances. At microwave frequencies, the global brightness temperature for oxygen sounding channels between 54 to $59 \mathrm{GHz}$ can be accurately simulated due to our knowledge of the oxygen absorption spectrum. It is also due to the fact that radiation for these channels originates mainly from the atmosphere, not the surface. As a result, at oxygen channels of 54.4, 55.5, 57.3, and $59.4 \mathrm{GHz}$, the simulation accuracy is mainly dependent on the temperature accuracy in regions where the weighting function peaks. For example, an error of $1 \mathrm{~K}$ in physical temperature where the weighting function peaks can result in an error of $0.1 \mathrm{~K}$ in simulated brightness temperature [1]. In this study, the Community RTM developed by the Joint Center for Satellite Data Assimilation (JCSDA) [12] is employed to simulate the brightness temperatures at SSMIS channels using the absorption model developed by Rosenkranz [13]. Inputs of the absorption model consist of temperature and water vapor profiles obtained from the global data assimilation system (GDAS) analysis by the National Center for Environmental Prediction (NCEP). The performance of the GDAS analysis products is continuously monitored by the NCEP Environmental Modeling Center (http://www.emc.ncep.noaa.gov/modelperf/). The root mean square error of GDAS temperature profiles against radiosonde measurements below $10 \mathrm{mb}$ is smaller than $2 \mathrm{~K}$, which results in an uncertainty of about $0.2 \mathrm{~K}$ in simulations from 54 to $59 \mathrm{GHz}$. Initially, the errors in simulated brightness temperatures are significantly less affected by the errors in GDAS water vapor profile because they are not sensitive to water vapor absorption. Also, the effects of cloud and rain are not taken into account in these simulations due to the lack of information. This is reasonable for the 54.4, 55.5, 57.3, and $59.4 \mathrm{GHz}$ channels because their weighting functions typically peak above the absorption layer that consists of clouds and precipitation. Thus, any bias beyond an uncertainty of around 0.2 (resulting from the temperature profile) can be attributed to calibration errors. Note that the brightness temperatures for F16 SSMIS instruments used in this study are converted from their antenna temperatures using the coefficients provided by the instrument manufacturer to NRL.

Shown in Figure 2 is the longitudinal mean difference between observed and simulated antenna temperatures for F-16 SSMIS $54.4 \mathrm{GHz}$ on March 20, 2005. For a direct comparison between simulation and observation, the simulated brightness temperatures have been converted hereinafter to the antenna temperatures. $T_{\mathrm{ARM}}$ is the arm 
temperature of the main reflector (more explanations are given in Section 2.3). Results in Figure 2 show that antenna brightness temperature biases have strong geographical dependence along with obvious reductions over several latitudinal zones labeled as "I V." Similar features are observed at 55.5, 57.3, and $59.4 \mathrm{GHz}$ channels (referring to Figures 8 and 10). After this analysis was extended to other days throughout the seasons, we found similar conclusions except for their variable magnitudes with regions (refer to Figure 10(b)). At other LAS channels, brightness temperatures over cloud-free oceans can be accurately simulated because of our knowledge of ocean emissivity [14]. Antenna temperature differences between the SSMIS observations and simulations at 50.3,52.8, and $53.6 \mathrm{GHz}$ are thus investigated over cloud-free oceans. Results show that the differences at these three channels have similar features to the above four sounding channels except for slightly distinctive magnitudes (figures are omitted here).

These geographically and seasonally dependent biases in SSMIS radiances at the LAS channels are considered as anomalies because we normally anticipate the bias for these sounding channels to be relatively small and independent of location and season. Root causes of the anomalies had been investigated which resulted from thermal emission by the SSMIS main reflector and solar intrusion on warm calibration target $[3,7,9,10]$, as briefly described in Sections 2.3 and 2.4 correspondingly.

2.3. Thermal Emission from SSMIS Main Reflector. The SSMIS antenna consists of an offset parabolic main reflector and array of feedhorns that work in concert to produce the antenna power patterns by a combination of the radiation patterns of the feedhorn and the main reflector. As the antenna views earth, brightness temperature of the earth scene incident upon the antenna is received and spatially filtered by the antenna to form antenna temperature $\left(T_{A}\right)$ at the input of the feedhorn. For a perfect reflector, this antenna temperature is only an integral of the scene brightness temperature distribution incident on the main reflector and the co- and cross-polarized far-field antenna power patterns [15]. For an emissive reflector, the radiance arrived at the feedhorn becomes $\left(1-\varepsilon_{R}\right) T_{A}$, where $\varepsilon_{R}$ denotes the emissivity of the main reflector.

Ideally, the reflector should be lossless, that is, its reflectivity is unity and its emissivity is zero. However, according to the SSMIS instrument calibration team, the F16 SSMIS main reflector is not a perfect reflector due to impurities in the reflector coating such that its emissivity could vary from 0.01 to 0.04 for frequencies between 19 and $183 \mathrm{GHz}$, respectively $[3,10]$. Furthermore, the main reflector is heated periodically by incident solar radiation and emits a variable radiation with space and time. As a result, the "extra" radiation comes at the expense of reduced radiation from the scene reflected into the feedhorns due to the reduced efficiency of the reflecting surface (i.e., $\varepsilon_{R} T_{R}$ ) where $T_{R}$ denotes the effective reflector temperature which is based on the feed illumination convolved with the temperature field of the reflector. In the rest of the paper, the effective reflector temperature is simply named to be reflector temperature. Therefore, the antenna temperature focused on the feedhorn including the main reflector emission contribution is given by

$$
T_{A}^{\prime}=\left(1-\varepsilon_{R}\right) T_{A}+\varepsilon_{R} T_{R}
$$

where the channel frequency and polarization of each variable are omitted for simplicity. However (1) is rewritten to be

$$
T_{A}^{\prime}=T_{A}+\varepsilon_{R}\left(T_{R}-T_{A}\right)
$$

which implies that the antenna emission $\varepsilon_{R}\left(T_{R}-T_{A}\right)$ must be accounted for in order to obtain the antenna temperature of the earth scene.

The magnitude of this antenna emission is linearly proportional to the reflector emissivity and the difference between the reflector temperature and the antenna temperature. At the current stage, a set of SSMIS emissivty for frequencies between 19 and $183 \mathrm{GHz}$ was simulated using the NRL multilayer antenna model [3, 10]. The reflector temperature is not provided in F-16. Instead, there is a thermometer mounted on the antenna arm to measure the arm temperature named as $T_{\mathrm{ARM}}$, as shown in Figure 2 . The correlation of the antenna temperature anomaly to $T_{\mathrm{ARM}}$ is observed except for several latitudinal zones where the solar intrusion results in certain discontinuity. However, the $T_{\mathrm{ARM}}$ does not respond as fast as the antenna temperature anomaly. This is caused by a slower response of the arm to solar heating than the reflector face since the thermometer measuring $T_{\mathrm{ARM}}$ is 0.3 meter away from the center of the main reflector. The difference between the arm and reflector temperatures is on the order of several tenths of Kelvin according to our retrieved reflector temperature provided in Section 3.2, which leads to at most $1 \mathrm{~K}$ underestimate of the antenna emission according to (2). Therefore, to derive reflector temperatures is a key issue for antenna emission correction.

2.4. Calibration Target Anomalies by Solar Intrusions. The SSMIS measurements are calibrated by passing the feedhorns through two fixed reference targets: a warm load and a cold space reflector. Let $C_{S}, C_{C}$, and $C_{W}$ denote the radiometric counts of earth scene, cold space, and warm load, respectively. Also, $T_{C}$ is the deep space cosmic background temperature, and $T_{W}$ is the PRT-measured brightness temperature of the warm load (PRT temperature). Generally, the scene counts are converted to the antenna temperature $T_{A}$ using the warm load and cold space counts along with their corresponding brightness temperatures,

$$
T_{A}=F\left(C_{S}, C_{W}, C_{C}, T_{W}, T_{C}\right)
$$

where $F$ is the calibration function.

Obviously, the accuracy of $T_{A}$ is affected by the selection of a calibration function $F$, which can be a linear or nonlinear function. The nonlinearity can result from an imperfect square law detector and/or intermediate frequency amplifier compression in the receiver subsystem. Generally speaking, 
the calibration accuracy for a microwave radiometer through a linear algorithm is not adequate [16-18]. However, the SSMIS nonlinear contribution at the LAS channels is not investigated in this study. Instead, $F$ in (3) is assumed to be linearly related to the antenna temperature and calibration parameters. Thus, we have

$$
T_{A}=T_{C}+S\left(C_{S}-C_{C}\right),
$$

where

$$
S=\frac{T_{W}-T_{C}}{C_{W}-C_{C}}
$$

Obviously, accuracy of antenna temperature relies on accuracy of calibration target information such as warm count, PRT temperature, and cold count. Unfortunately, soon after launch of the SSMIS, solar radiation impinges upon the surfaces of the warm load tines and results in rapid heating of warm load tines over several geometric scenarios [19]. Detailed analyses are referred to Swadley et al. [19] and omitted here. A direct result of this heating of warm load tines is a sudden short-term positive jump in warm counts which is named as warm count anomaly or $\Delta C_{W}\left(\Delta C_{W}\right.$ $>0$ ). Figure 3(a) displays the time series of warm counts at channel $4(54.4 \mathrm{GHz})$ for one orbit of observations in Figure 2. The warm count anomalies are clearly seen in the zones labeled as "I $\sim$ V," and each zone is accompanied with short-term jumps in warm counts whose maximum distributes between 20 and 60 counts.

However, this solar intrusion does not cause obvious temperature anomaly and gradient in three warm load PRT temperatures. Figure 3(b) displays the time series of three warm load PRT temperatures for the same orbit. More examples are given in Figures 4(a)-4(1), where the times series of three PRT temperatures are shown during two orbits of observations for one day each month in 2005. The maximum fluctuation in three PRT temperatures due to solar intrusion is only around $0.2 \mathrm{~K}$ and the affected region is also smaller than warm counts. According to the estimate of the channel gain (Figure 3(c)), there is a variation of about 37 counts per $1 \mathrm{~K}$. Thus, the magnitude and phase of fluctuations in $T_{W}$ do not match with these of the fluctuations in $C_{W}$. This phenomenon is attributed primarily to the thermal inertia of the warm load and the fact that the PRTs are imbedded deep enough inside the substrate of the warm load and not at the surface viewed by the radiometer where much of solar intrusion occurs. Note that the gradient among three PRT temperatures is very small (see Figures 3 (b) and 4). This feature is different from that in WindSat where a significant temperature gradient of more than $0.5 \mathrm{~K}$ exists among six PRT temperatures due to solar radiation [20].

Due to the aforementioned mismatch, according to calibration (4), an error in the antenna temperature (i.e., $\Delta T_{A}^{\mathrm{CAL}}$ ) may be caused, viz.,

$$
\begin{aligned}
& \Delta T_{A}^{\mathrm{CAL}}= \\
& \quad-\left[\frac{T_{A}-T_{C}}{C_{W}-C_{C}}\right] \Delta C_{W}-\left[\frac{T_{W}-T_{A}}{C_{W}-C_{C}}\right] \Delta C_{C}+\left[\frac{C_{S}-C_{C}}{C_{W}-C_{C}}\right] \Delta T_{W} .
\end{aligned}
$$

Table 2 displays the error in the antenna temperature due to warm count anomalies over several zones which are computed using the first term in (6). Results show that the solar intrusion anomaly in $C_{W}$ can impart up to a maximum of $1.1 \mathrm{~K}$ depression in antenna temperatures near the center of the intrusion period, while the error due to PRT temperature anomaly is only about $0.1 \mathrm{~K}$ (not shown in table). This analysis is further confirmed by consistency in magnitudes and positions of those warm count anomalycaused errors in antenna temperatures with the antenna temperature differences over the same zones in Figure 2. Since the anomaly of about 10 counts was sometimes detected in the cold space count for some channels (figures are omitted), the effect of cold count anomaly $\left(\Delta C_{C}\right)$ is included in (6).

A similar analysis can be applied to other LAS channels. Besides the anomaly in $54.4 \mathrm{GHz}$, the anomaly of warm counts also occurs at other LAS channels. Usually, the phase of the solar intrusion on the warm load is dependent on channels. It is the location of the solar illumination on the warm load tines and the relative position and timing of the warm load calibration observations with respect to the series of feedhorns that causes the phasing. However, for the LAS channels, the antenna temperatures arrive at the feedhorn at nearly the same time, that is, the phase of the anomaly is similar. Figure 3(d) displays the times series of the warm counts at all LAS channels (i.e., channels 1-7). These results indicate that while the magnitude of each anomaly is variable with channel, the phase of each channel anomaly is similar because their time difference is small.

Furthermore, the features of the warm count anomalies are investigated for various seasons. Figure 3(e) displays time series of $C_{W}$ of one orbit of observations at $54.4 \mathrm{GHz}$ for the first day of each month throughout the year of 2005. It is observed that the solar intrusion to warm load always occurs and the resulted warm count anomaly magnitude changes gradually with season. However, several common features are noted from the warm count anomaly distribution. The warm count anomaly lasts from 5 to 15 minutes every time the solar intrusion occurs, and the locations of five zones are correlated to the positions of the maximum/minimum warm counts in the time domain, that is, two of them occur near $C_{W}$ maximum; two prior to the $C_{W}$ minimum and one after the $C_{W}$ minimum. In addition, the five solar intrusion zones affect $30-40 \%$ of the entire orbital observations.

Therefore, the radiance anomalies at the LAS channels are characterized by both the antenna emission and warm count anomaly and display regionally and seasonally dependent features. These complicated features significantly increase complexity and difficulty of accurate corrections for such variable anomalies. However, those erroneous SSMIS radiances at the LAS channels are inappropriate for assimilation into the numerical weather prediction model. The reliable identification and correction of those anomalies per orbit are thus mandatory for the utilization of SSMIS data in weather prediction models. To remove these calibration anomalies in F16, three independent preprocessors for SSMIS radiances were studied from 2005 on in NRL, UKMET, and NOAA National Environmental 


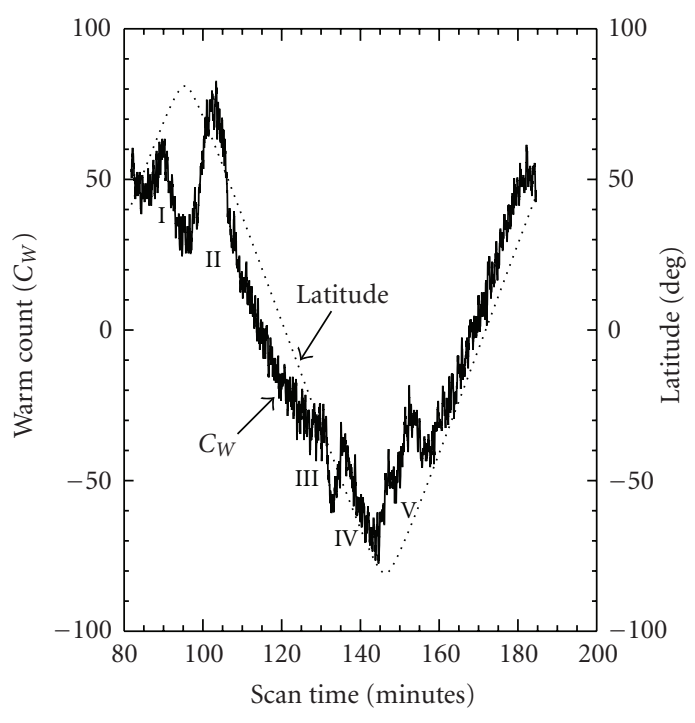

(a)

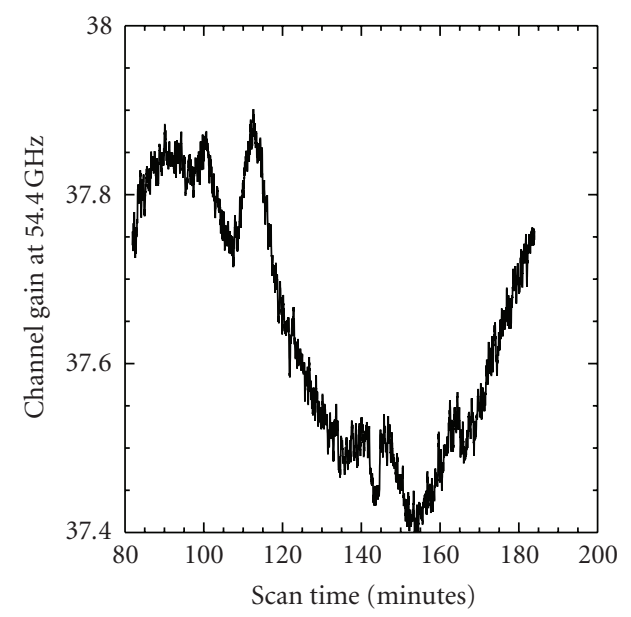

(c)

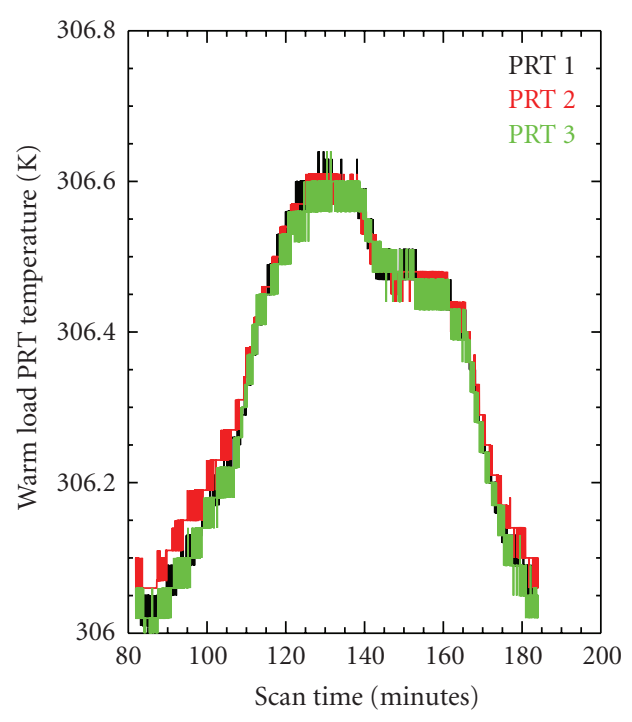

(b)

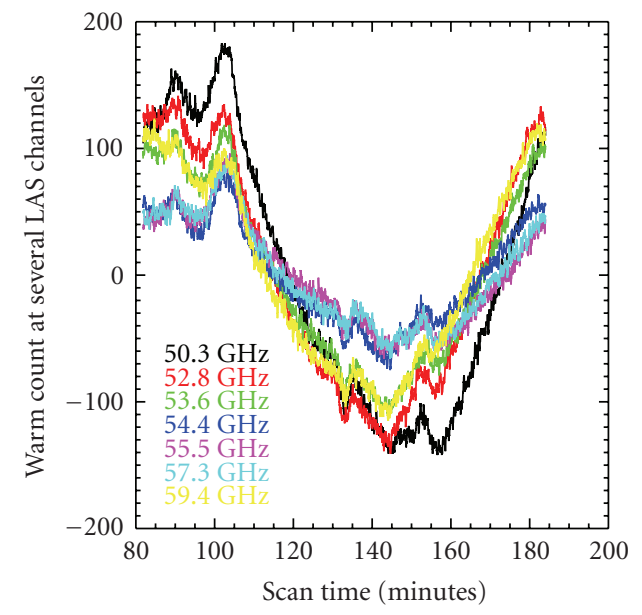

(d)

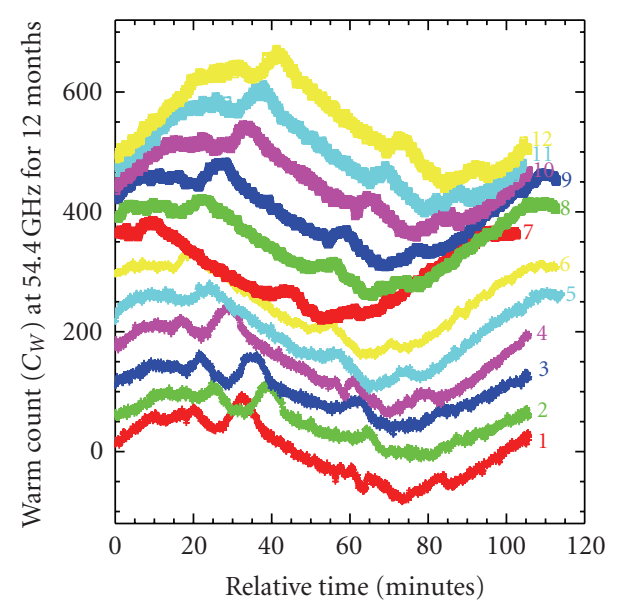

(e)

FIgURE 3: Time series of $C_{W}, T_{W}$, gain, during one orbit of observations, on March 20, 2005 for (a) (d) and on the first day of twelve months 2005 for (e), where a constant (orbital mean of corresponding counts) has been subtracted from the warm count to emphasize characteristics of warm count anomaly. (a) $C_{W}$ at $54.4 \mathrm{GHz}$. (b) $T_{W}$. (c) gain at $54.4 \mathrm{GHz}$. (d) $C_{W}$ at 50.3, 52.8, 53.6, 54.4, 55.5, 57.3, 59.4 GHz. (e) Time series of $C_{W}$ at $54.4 \mathrm{GHz}$ during one orbit in twelve months in 2005 , where the numbers from 1 to 12 denote the month of data. 


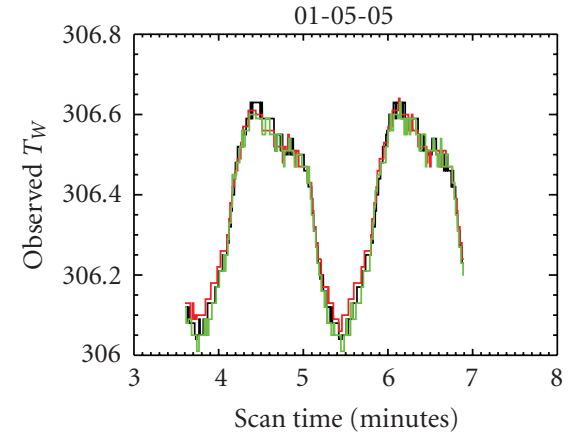

(a)

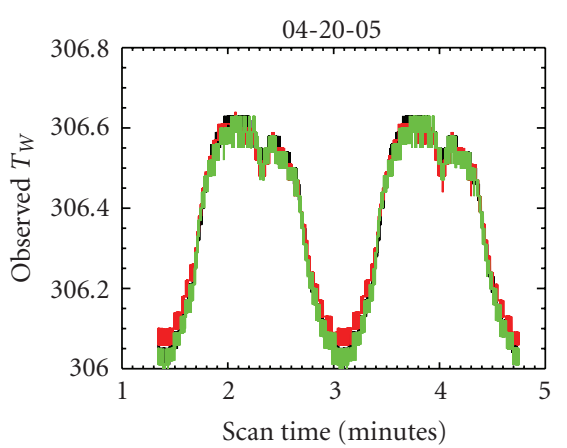

(d)

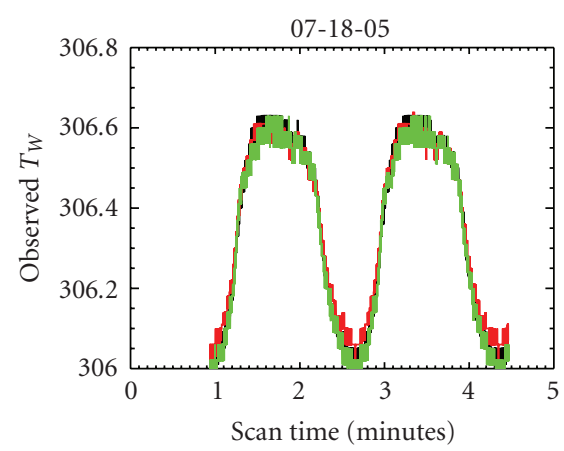

(g)

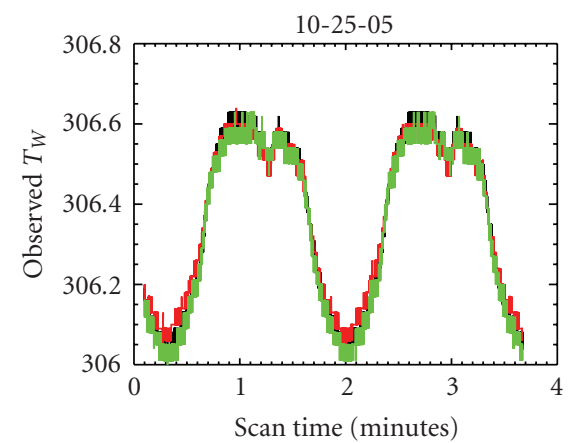

(j)

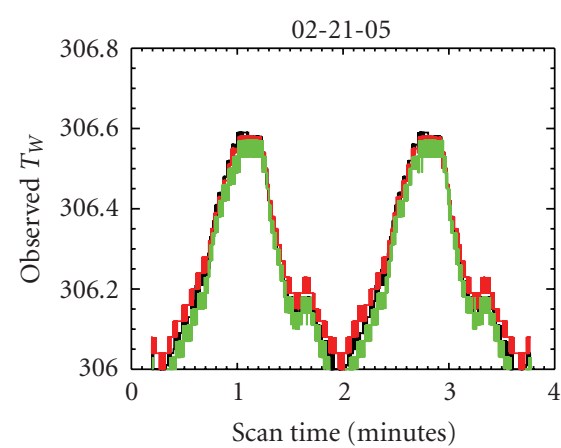

(b)

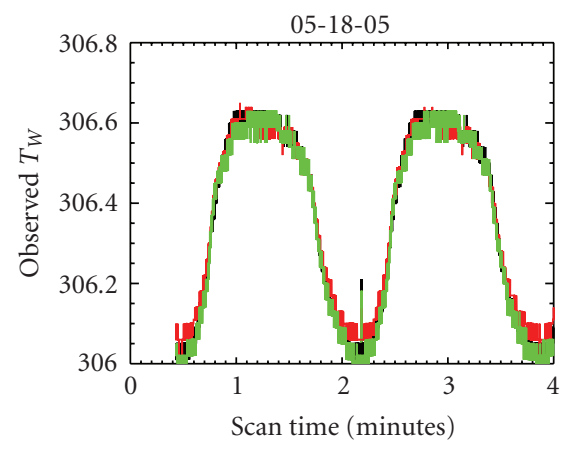

(e)

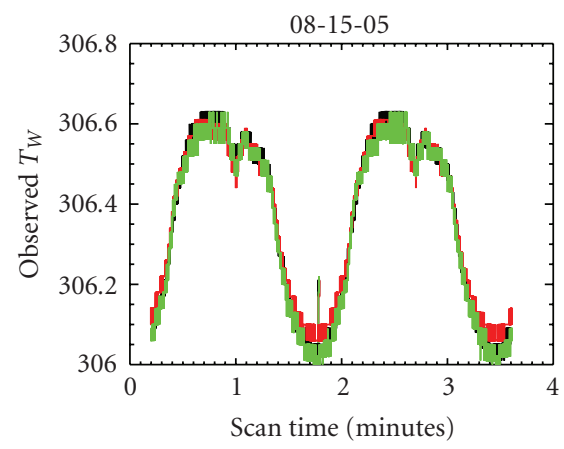

(h)

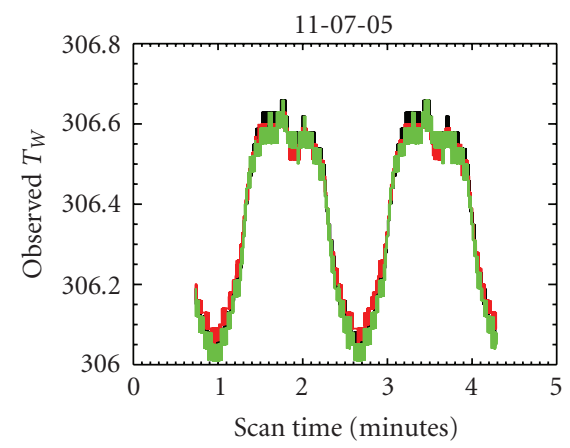

(k)

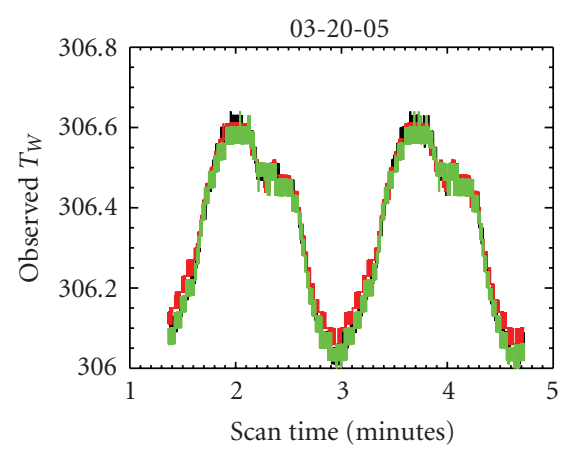

(c)

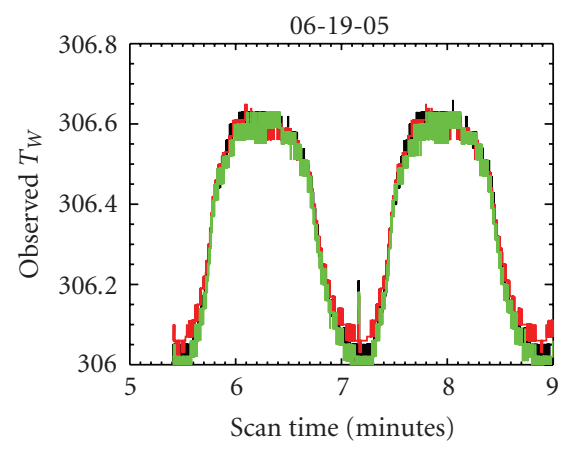

(f)

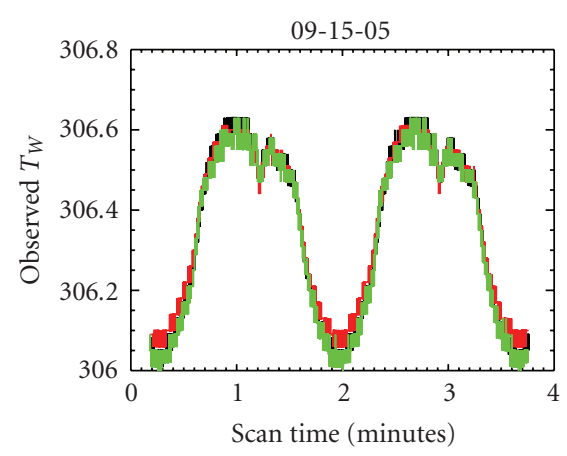

(i)

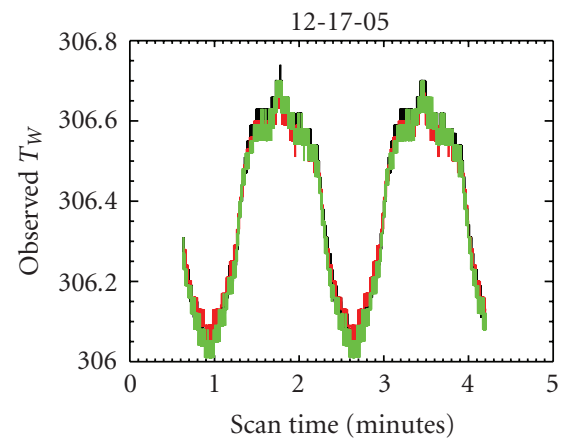

(1)

Figure 4: Time series of three PRT temperatures $\left(T_{W}\right)$ during two orbits of observations for twelve days in 2005, where the black, red, and green represent three PRT temperatures. (a) January 5. (b) February 21. (c) March 20. (d) April 20. (e) May 18. (f) June 19. (g) July 18. (h) August 15. (i) September 15. (j) October 25. (k) November 7. (l) December 17. 
TABLE 2: Warm load count anomaly zone identified from SSMIS data March 20, 2005. $\beta_{L} \sim \beta_{R}$ denotes the latitudes of each anomaly zone width in the left and right sides, respectively. $\left(\Delta C_{W}\right)_{\max }$ is the maximum warm load count anomaly for each zone and is estimatedaccording to Figure 3(a). $\left(\Delta T_{A}^{\mathrm{CAL}}\right)_{\max }$ is the error in SSMIS radiance corresponding to $\left(\Delta C_{W}\right)_{\max }$, which is determined using the first term in (6), (i.e., $\left.-\left(T_{A}-T_{C}\right) /\left(C_{W}-C_{C}\right) \Delta C_{W}\right)$.

\begin{tabular}{lccccc}
\hline Names & Zone 1 (ascending) & Zone 2 (descending) & Zone 3 (descending) & Zone 4 (descending) & Zone 5 (ascending) \\
\hline$\beta_{L} \sim \beta_{R}$ & $40^{\circ} \mathrm{N} \sim 80^{\circ} \mathrm{N}$ & $56^{\circ} \mathrm{N} \sim 80^{\circ} \mathrm{N}$ & $3^{\circ} \mathrm{S} \sim 42^{\circ} \mathrm{S}$ & $42^{\circ} \mathrm{S} \sim 74^{\circ} \mathrm{S}$ & $56^{\circ} \mathrm{S} \sim 74^{\circ} \mathrm{S}$ \\
Time (minute) & 8 & 15 & 12 & 13 & 15 \\
$\left(\Delta C_{W}\right)_{\max }$ & 30 & 60 & 20 & -0.6 & 20 \\
$\left(\Delta T_{A}^{\mathrm{CAL}}\right)_{\max }(\mathrm{K})$ & -0.6 & -1.1 & -0.4 & -0.6 \\
\hline
\end{tabular}

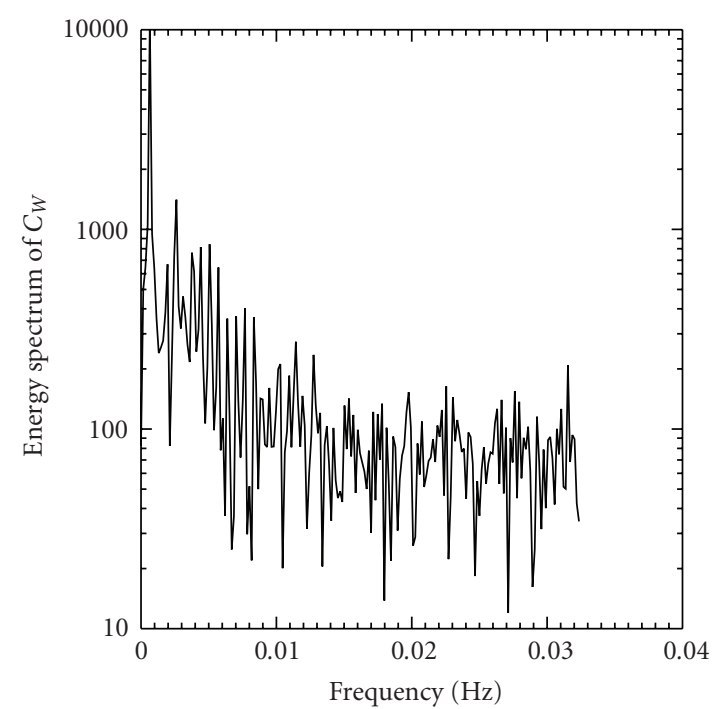

(a)

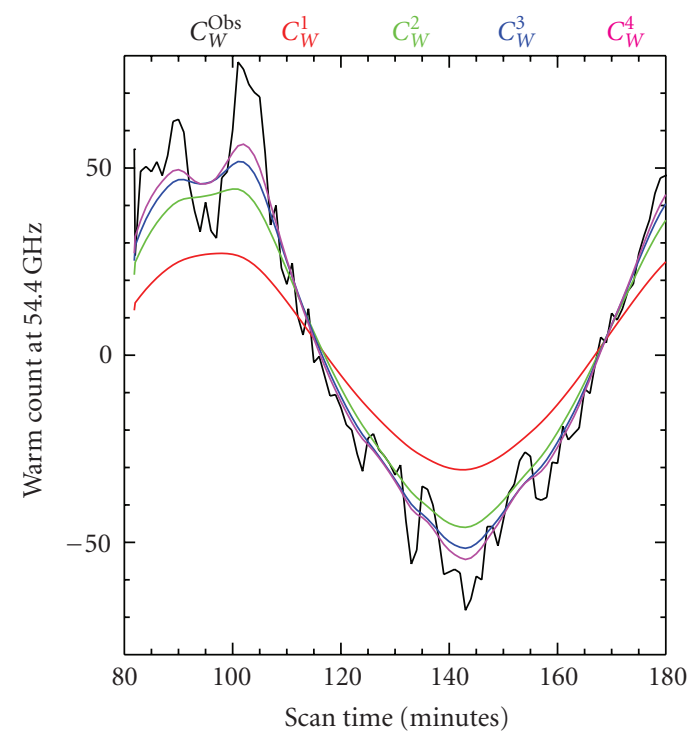

(b)

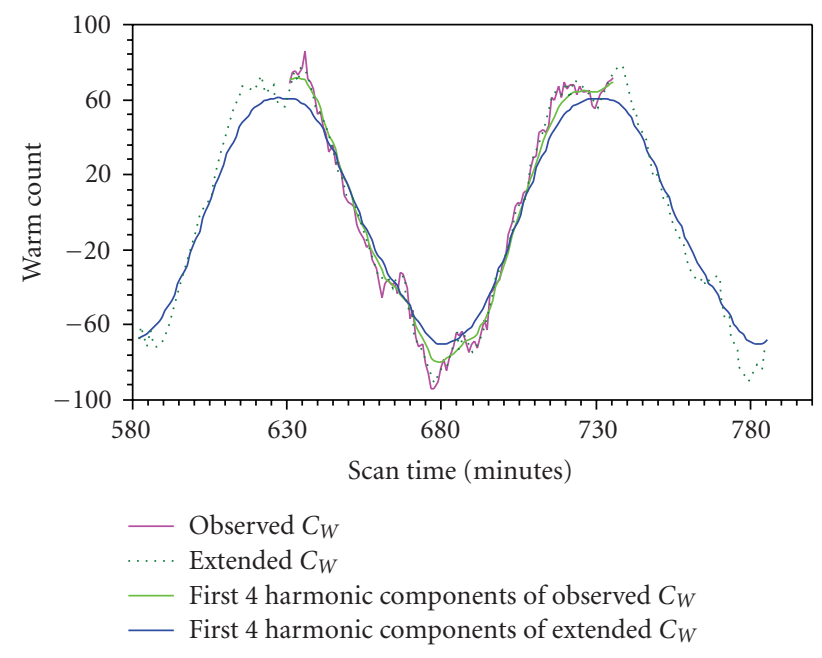

(c)

FIgURE 5: (a) FFT energy spectrum of $C_{W}^{\text {Obs }}$ at channel 4 (54.4 GHz) during one orbit of observations on March 20, 2006. (b) Time series of $C_{W}^{\mathrm{Obs}}, C_{W}^{1} \sim C_{W}^{4}$ at $54.4 \mathrm{GHz}$ for the same orbit as in (a). (c) Comparisons of $C_{W}^{\mathrm{Obs}}, C_{W}^{\mathrm{Ext}}$ and its periodically extended warm counts at $54.4 \mathrm{GHz}$. In the figures, an orbital mean of corresponding counts has been subtracted from the warm counts in each orbit of observations. 


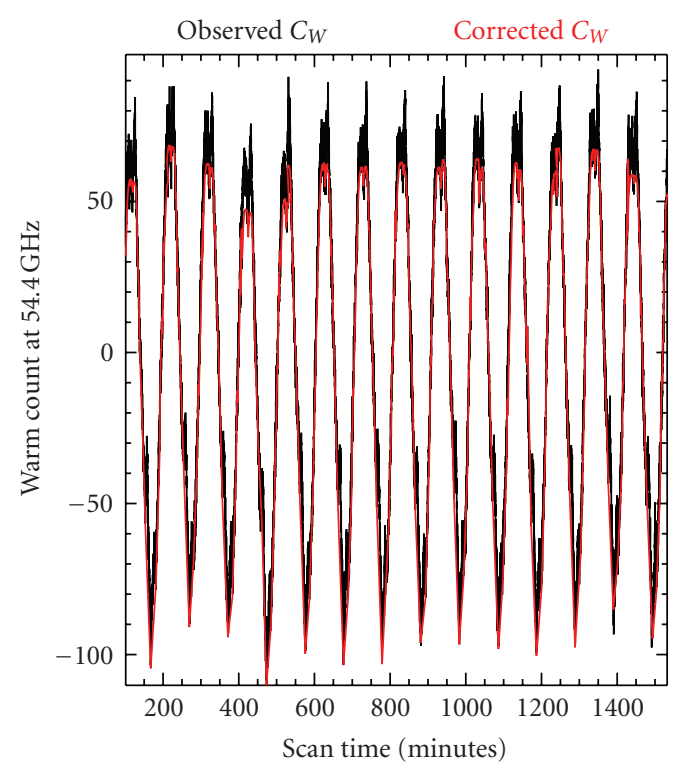

Figure 6: Time series of $C_{W}^{\mathrm{Obs}}$ and corresponding $C_{W}^{\mathrm{Cor}}$ at $54.4 \mathrm{GHz}$ for all orbits on August 15, 2006. In the figures, an orbital mean of corresponding counts has been subtracted from the warm counts in each orbit of observations.

Satellite, Data, and Information Service (NESDIS) and are currently available $[8,19,21]$. It is probably fair to say that none of these preprocessors would be described by their authors as definitive but represent their best efforts to date to correct the instrumental biases evident in F-16 SSMIS data. In what follows, the NOAA NESDIS preprocessor for SSMIS radiances developed by Yan et al. [8] is introduced.

\section{Methodology of F16 SSMIS Radiance Anomaly Correction Algorithm}

3.1. Correction of Calibration Warm Count Anomaly. Correction of calibration warm count anomaly relies primarily on fast Fourier transform (FFT) analysis of warm counts due to a distinctive feature of warm count anomaly from major components of warm counts in frequency domain. The solar intrusion-caused anomaly is readily evident in the time series of warm counts, where several areas of sudden short-term positive jumps are observed in warm counts. In the domain of frequency, those anomalies represent a set of higher-frequency components superimposed on $C_{W}$ in comparison to its primary frequency component. Figure 5(a) displays the energy spectrum of observed warm counts $\left(C_{W}^{\text {Obs }}\right)$ at channel 4 for an orbit (around 102 minutes), while Figure 5(b) displays time series of both $C_{W}^{\mathrm{Obs}}$ and a few orders of harmonic components (i.e., $C_{W}^{1} \sim C_{W}^{4}$ ), which are calculated using FFT analysis. According to SSMIS satellite period (about 102 minutes), the primary frequency component of $C_{W}^{\mathrm{Obs}}$ is approximately $1 / 102\left(\mathrm{~min}^{-1}\right)$ or $0.00016 \mathrm{~Hz}$. Thus, the solar intrusion-caused components are distributed primarily beyond the frequency range of the first few harmonic components of the FFT spectrum of $C_{W}^{\mathrm{Obs}}$.
By assessing the antenna temperature differences using the reconstructed warm count time series, it is found that the first four or five harmonic components can capture the major features of the solar intrusion-corrected $C_{W}$ over the first two (strong) solar intrusion-contaminated zones in Figure 5(b), while the first six or higher harmonic components may be needed to correct warm count anomaly over zones 35 where the solar intrusion is relatively weak. Therefore, initially, we can filter out major high-frequency components in $C_{W}$ related to solar contamination by replacing $C_{W}^{\mathrm{Obs}}$ with its respective order of harmonic components, and further construct solar intrusion-corrected warm counts $\left(C_{W}^{\mathrm{Cor}}\right)$. Finally, in principle, regions where $\Delta C_{W}\left(C_{W}^{\mathrm{Obs}}-C_{W}^{\mathrm{Cor}}\right)$ exceeds a certain threshold are identified and the calibration correction is calculated using (6) there.

In this process, it is important to reduce truncating effect on FFT spectrum of SSMIS data (e.g., $C_{W}^{\mathrm{Obs}}$ ). When we use FFT to measure the frequency content of a time series of data, we have to base the analysis on a finite set of data (e.g., one orbit of observations in our study). The FFT transform assumes that the finite dataset is one period of a periodic signal, that is, the two endpoints of the data are interpreted as though they were connected together. However, satellite-measured data in an orbit may deviate from this feature. As a result, the finiteness of one orbit of data may result in a truncated waveform nearby two edges of time-finite signal. In this study, this effect can be significantly reduced by a periodical extension of the observed data, that is, artificially extending observations from one orbit (approximate one period) to about two orbits (approximate two periods). According to our analysis, the warm counts in SSMIS observations show good stability and consistency from one orbit to its neighboring orbits. This allows us to extend original warm counts $\left(C_{W}^{\mathrm{Obs}}\right)$ into a new dataset $\left(C_{W}^{\mathrm{Ext}}\right)$ of about two periods (orbits), where the first half orbit of data in $C_{W}^{\text {Ext }}$ is constructed by using the second half orbit of $C_{W}^{\mathrm{Obs}}$, the last half orbit of data in $C_{W}^{\mathrm{Ext}}$ is constructed by using the first half orbit of $C_{W}^{\text {Obs }}$, the rest of $C_{W}^{\text {Ext }}$ consists of the observed warm counts. As a result, the FFT analysis is applied to a periodically extended $C_{W}^{\text {Ext }}$ instead of $C_{W}^{\text {Obs }}$. In this way, the truncating effect occurs only during the extended data part instead of the original data part, as shown in Figure 5(c). Thus, the spectral characteristics of $C_{W}^{\mathrm{Obs}}$ with less truncating effect can be extracted from the FFT spectrum of artificially extending warm counts $\left(C_{W}^{\text {Ext }}\right)$.

In addition, the quality check of the reconstructed warm counts is also important to avoid over-correcting the effect of solar intrusions on warm counts. It has been known that solar intrusions are always related to regions nearby the positions of the maximum/minimum of $C_{W}^{\mathrm{Obs}}$ and each intrusion occurs between 5 and 15 minutes in different seasons, based on our statistical analysis throughout one year of data (Figure 3(e)). This feature may help identify rough locations of several solar intrusions on warm counts in different season. Then, the reconstructed warm counts only replace the observed warm counts over these regions. Figure 6 shows $C_{W}^{\mathrm{Obs}}$ and corresponding $C_{W}^{\mathrm{Cor}}$ at channel 4 for all orbits of observations on August 15, 2006, where $C_{W}^{\text {Cor }}$ in each orbit is generated by applying the above procedures. 
The reconstructed warm counts in the figure effectively correct most of the anomalies from solar intrusion. A similar analysis can be applied to other observations and channels in a year. By assessing corresponding antenna temperature differences based on the reconstructed warm counts, the residual antenna temperature errors are usually smaller than $0.1 \mathrm{~K}$ (the figures are omitted).

3.2. Removal of Antenna Emission. From the linear calibration equation (4) and the reconstructed calibration counts, the antenna temperatures can be obtained. This antenna temperature $\left(T_{A}^{\prime}\right)$, however, includes the contribution of the antenna emission. Theoretically, we can derive the antenna temperature from the earth scene's radiance by removing antenna emission using (2), that is,

$$
T_{A}=\frac{T_{A}^{\prime}-\varepsilon_{R} T_{R}}{1-\varepsilon_{R}} .
$$

Obviously, the variables required to perform this calculation are the reflector temperature and emissivity. Unfortunately, neither of them is available from measurements. For the reflector emissivity, a set of reflector emissivity at frequencies from $19.35 \mathrm{GHz}$ to $183 \mathrm{GHz}$ are given from the NRL emissivity model simulations. With this reflector emissivity, the remaining issue is to find reflector temperature $\left(T_{R}\right)$.

Estimate of reflector temperatures are made from the arm temperature of the main reflector (i.e., $T_{\mathrm{ARM}}$ ). As discussed in Section 2, $T_{\mathrm{ARM}}$ is an important indicator because it displays a similar orbital feature to the radiance difference (antenna emission) except for its response lag to solar heating (see Figure 2). So, the reflector temperature can be calculated in terms of $T_{\mathrm{ARM}}$ with a proper adjustment $[7,9]$, that is,

$$
T_{R}\left(t_{i}\right)=T_{\mathrm{ARM}}\left(t_{i}\right)+\Delta T_{R}\left(t_{i}\right),
$$

where $\Delta T_{R}$ represents an adjustment corresponding to the difference between $T_{R}$ and $T_{\mathrm{ARM}}$ due to the response lag of the arm to solar heating, $t$ the time of each scan line corresponding to a specific latitude, and the subscript " $i$ " the $i$ th scan line per orbit.

In this study, $\Delta T_{R}$ is determined by using a group of polynomial expressions for latitude. The fitting coefficients of polynomial expressions are derived from the training datasets of $T_{R}$ (and $T_{\mathrm{ARM}}$ ), and this $T_{R}$ is generated by solving (7) with the RTM simulations, that is,

$$
T_{R, i \mathrm{ch}}=\frac{T_{A, i \mathrm{ch}}^{\prime}-\left(1-\varepsilon_{R, i \mathrm{ch}}\right) T_{A, i \mathrm{ch}}}{\varepsilon_{R, i \mathrm{ch}}}
$$

where $\varepsilon_{R, i c h}$ is the emissivity at the $i$ th channel, and $i=4,5,6,7$, corresponding for $54.4,55.5,57.3,59.4 \mathrm{GHz}$. However, $T_{A}$ in (9) is obtained approximately from the RTM simulated antenna temperature.

After the training dataset of the retrieved $T_{R}$ and observed $T_{\mathrm{ARM}}$ is obtained, $\Delta T_{R}$ is further derived as an equation in terms of latitude $(\beta)$, which is expressed as

$$
\Delta T_{R, i \mathrm{ch}}=a_{0 i}+\sum_{j=1}^{N} a_{j i} \beta .
$$

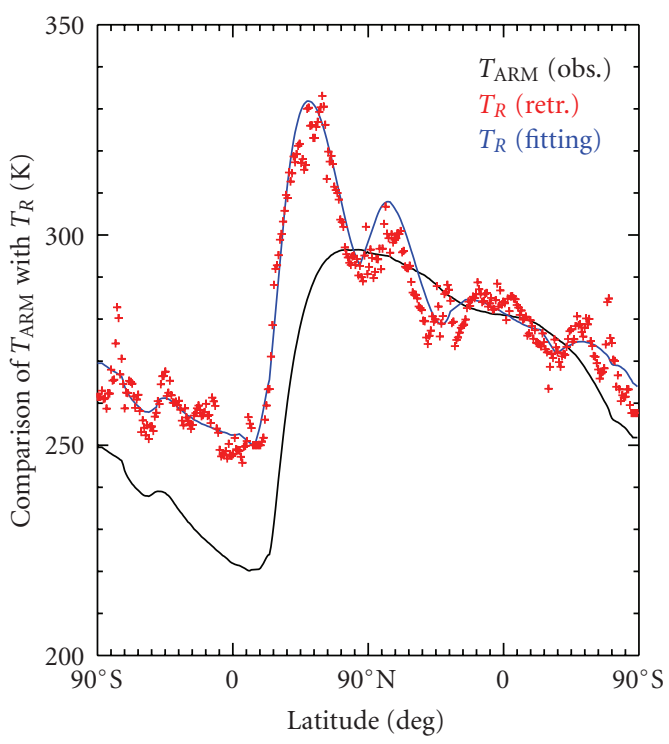

Figure 7: Comparisons of the observed arm temperature, the retrieved and fitted reflector temperatures at $54.4 \mathrm{GHz}$ during one orbit of observations on March 20, 2006.

As an example, Figure 7 shows the reflector arm temperature, the retrieved and fitted reflector temperatures at $54.4 \mathrm{GHz}$, on March 20, 2006, where the retrieved reflector temperature is calculated using (9) and the fitted reflector temperature is calculated using (8) and (10). It is found that the retrieved reflector temperature can be fitted well from (8) and (10). In comparison to the observed $T_{\mathrm{ARM}}$, the $T_{R}$ at channel 4 responds to the solar heating faster than $T_{\mathrm{ARM}}$ and the most significant adjustment (i.e., a maximum $\Delta T_{R}$ ) is made when the spacecraft is out of the Earth's eclipse (around $20^{\circ} \mathrm{N}$ in the ascending node). Consequently, the reflector temperature captures more closely the trend of the bias than the arm temperature. The reflector temperature does show a significant orbital cycle where it varies between 220 and $330 \mathrm{~K}$. This large variation in $T_{R}(100 \mathrm{~K})$ can explain a maximum error of $2 \mathrm{~K}$ in SSMIS antenna temperature at oxygen channels. So-estimated emission magnitude at $54.4 \mathrm{GHz}$ agrees well with the observed anomaly.

According to the results of the reflector temperatures at the four channels retrieved using (9), some similar orbital features are observed from the four retrieved reflector temperatures (the figures are omitted here) although their magnitudes vary slightly with channel with a difference of about $10 \mathrm{~K}$. For simplicity, the reflector temperatures at the other three channels are produced from the reconstructed reflector temperatures at channel 4 with some constant offsets from 10 to $15 \mathrm{~K}$. Currently, we are unable to accurately simulate the global brightness temperatures at channels $1 \sim 3$ due to lack in information of accurate clouds and land surface emissivity. It has been known that the observations from channels $1 \sim 5$ are received through the A4 antenna feed. Also, the difference in the retrieved reflector temperatures at the four channels between 4 and 7 is below $15 \mathrm{~K}$ over most of areas. So, in this study, the reflector 


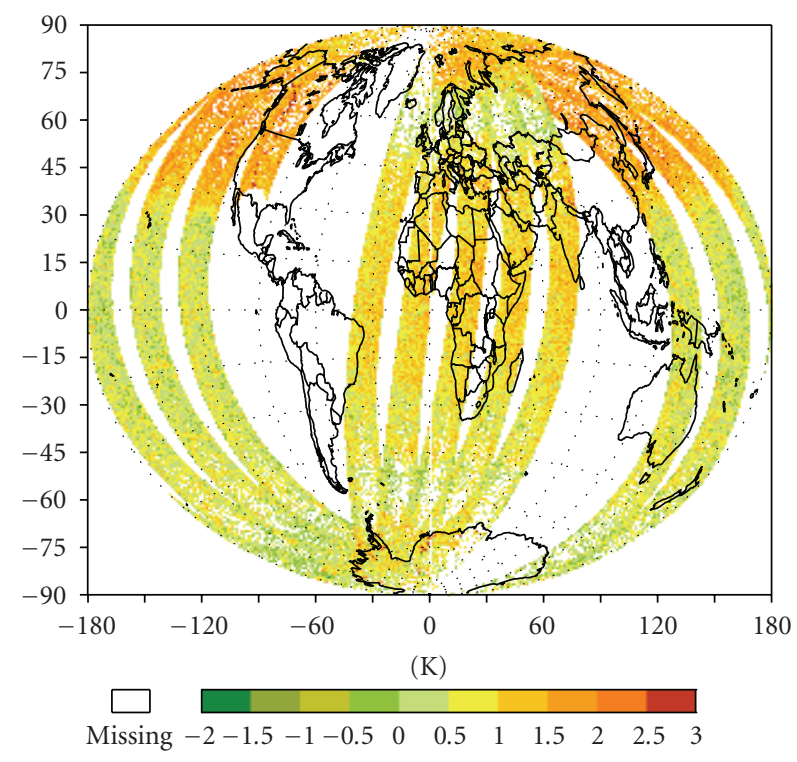

(a) SSMIS observations-simulations at $54.4 \mathrm{GHz}, 2006-03-20$

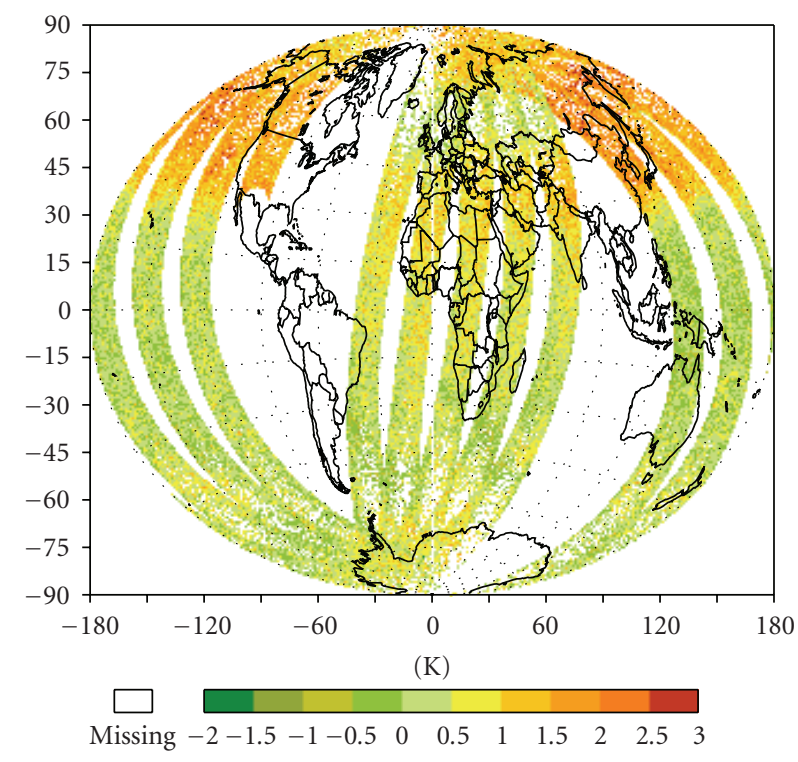

(b) SSMIS observations-simulations at $55.5 \mathrm{GHz}, 2006-03-20$

FIGURE 8: Global distribution of antenna temperature bias between SSMIS TDR measurements and radiative transfer simulations on March 20, 2006 for (a) $54.4 \mathrm{GHz}$, and (b) $55.5 \mathrm{GHz}$.

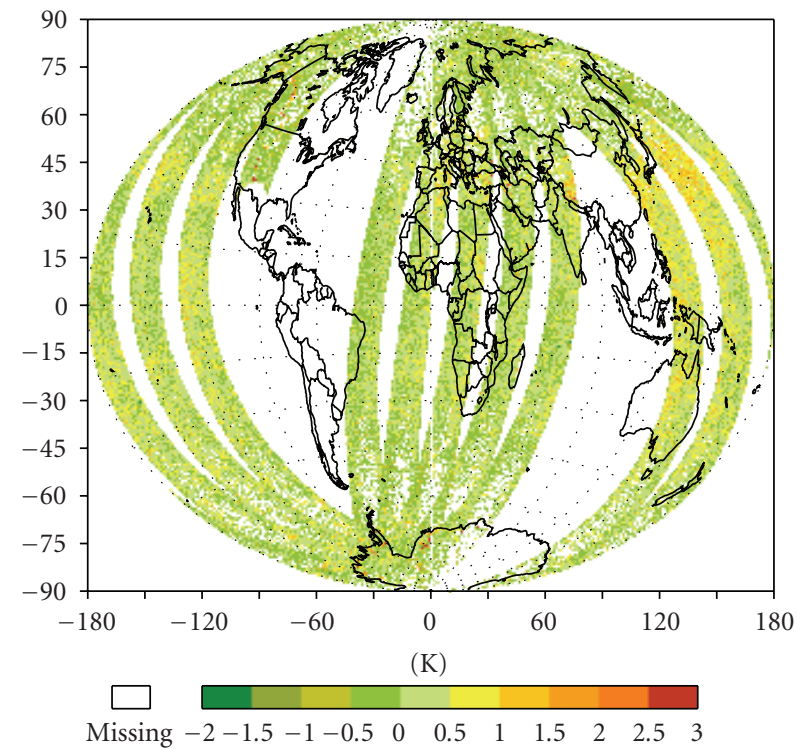

(a) Cal. SSMIS observations-simulations at $54.4 \mathrm{GHz}, 2006-03-20$

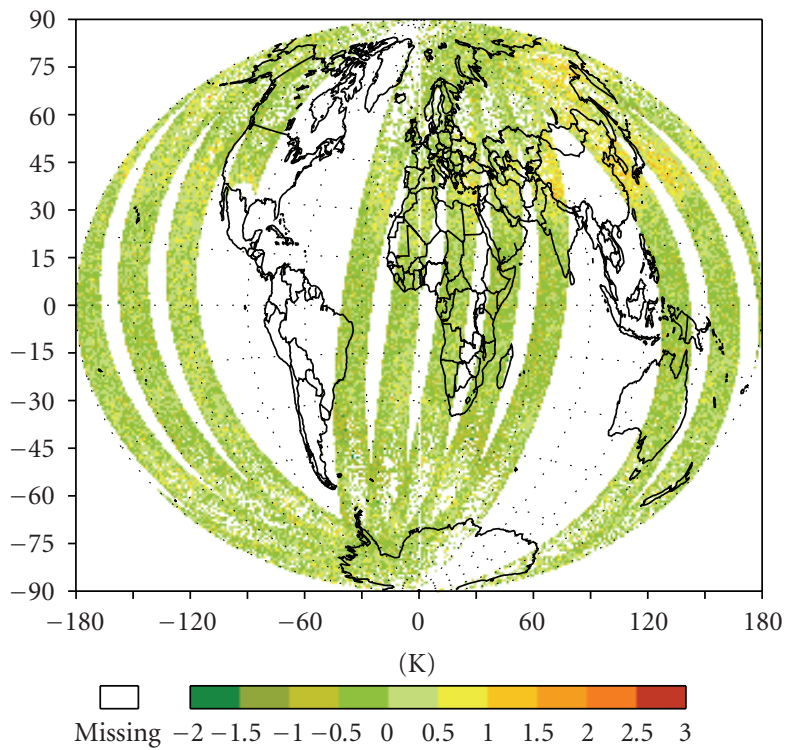

(b) Cal. SSMIS observations-simulations at $55.5 \mathrm{GHz}, 2006-03-20$

FIGURE 9: Global distributions of antenna temperature bias between F16 CTDR measurements and radiative transfer simulations where "Cal." denotes the calibrated antenna temperatures using the newly developed calibration algorithm for (a) $54.4 \mathrm{GHz}$ and (b) $55.5 \mathrm{GHz}$.

temperatures at channels $1 \sim 3$ are assumed to be equivalent approximately to that at channel 4 .

\section{Evaluations of SSMIS Anomaly Correction Algorithms}

4.1. Comparisons with RTM Simulations and AMSU-A Observations. Performance of the SSMIS antenna temperature anomaly correction algorithm is assessed using the observed and RTM-simulated brightness temperatures at all LAS channels. For demonstration, Figures $8(\mathrm{a})-8(\mathrm{~b})$ display the global distributions of the bias at 54.4 and $55.5 \mathrm{GHz}$ for March 20, 2006, which are computed using the SSMIS TDR data; Figures 9(a)-9(b) display the global distributions of the bias at the same frequencies, which are computed using the antenna temperatures after the SSMIS anomaly correction (known as CTDR). In comparison to results for SSMIS TDR data in Figures 8(a)-8(b), the antenna temperature biases caused by antenna emission and warm count anomaly 


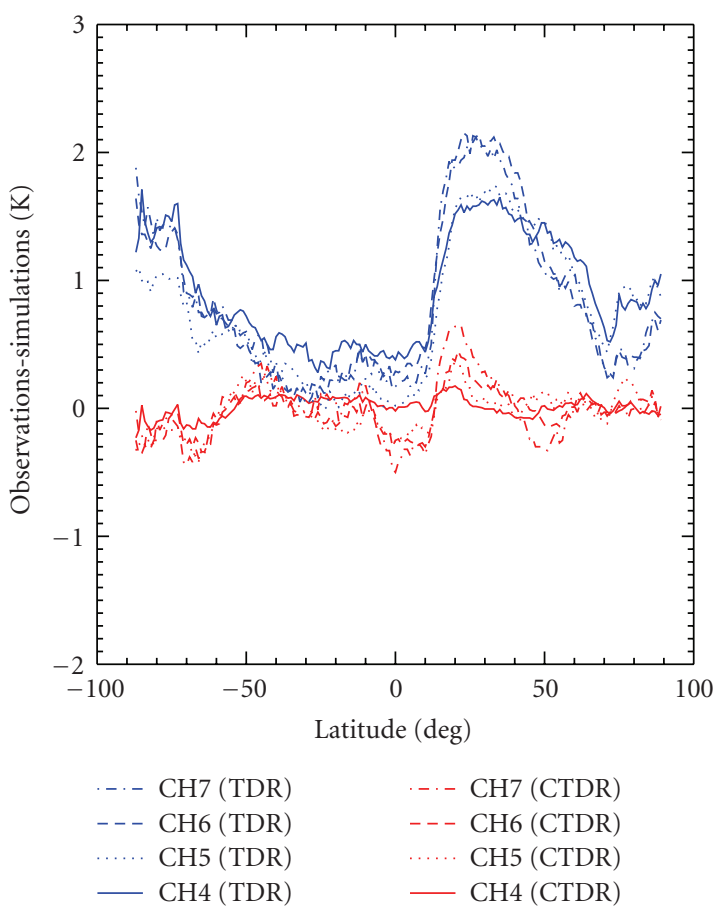

(a)

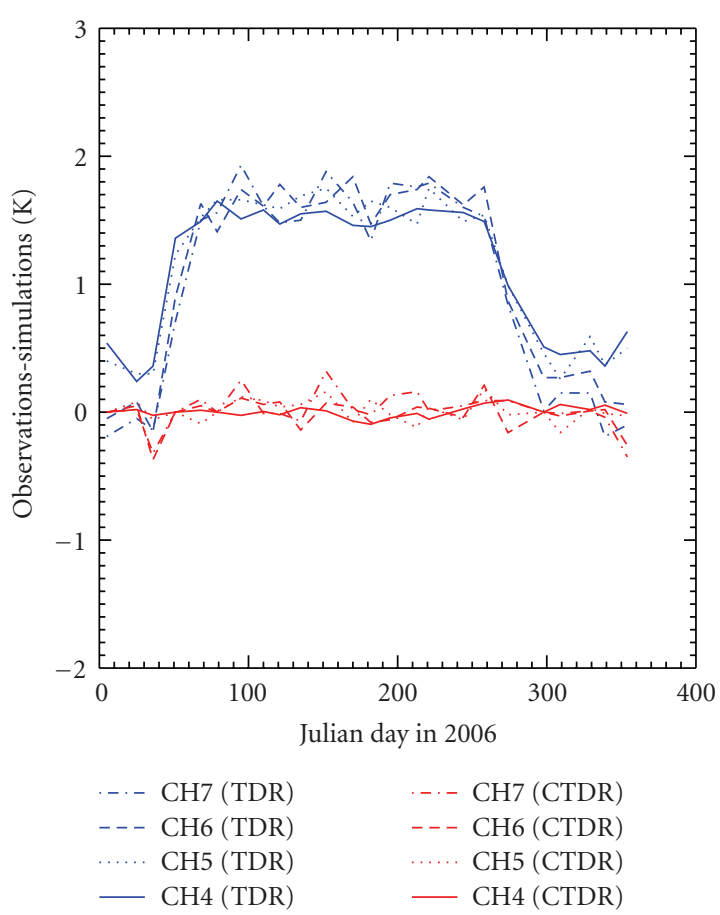

(b)

FIGURE 10: (a) Longitudinally averaged antenna temperature biases between F16 SSMIS TDR (CTDR) measurements and simulations at $54.4,55.5,57.3$, and $59.4 \mathrm{GHz}$ on June 19, 2006, and (b) time series of the longitudinally averaged antenna temperature biases at latitude of $50^{\circ} \mathrm{N}$. In the figures, red is for newly calibrated while blue for original TDR results.

are mostly corrected in Figures 9(a)-9(b). The absolute biases in CTDR data are typically smaller than $0.5 \mathrm{~K}$ over most of the global areas. A similar comparison may be applied to channels $1-3$, but the regions are limited to cloud-free oceans due to lack of accurate information about clouds and land emissivity. Results also show that antenna temperature differences by using CTDR data are typically within $0.5 \mathrm{~K}$ (figures are omitted). This conclusion is also valid for longer sets of SSMIS TDR data throughout 2006. As an example, Figure 10(a) shows the mean bias for a few SSMIS sounding channels plotted against latitude on June 19, 2006, whereas Figure 10(b) displays a time series of the mean bias at latitude of $50^{\circ} \mathrm{N}$ in descending node throughout 2006. Note that the antenna temperature anomalies at certain latitudes show a strong seasonal feature. For example, the antenna temperature anomalies at $50^{\circ} \mathrm{N}$ from March to September vary around $1.8 \mathrm{~K}$, but they gradually reduce to around $0.5 \mathrm{~K}$ beyond this period. Also, the gradual shifts in phase and magnitude of the biases are observed. This is presumably a result of the change in satellite to sun's position from season to season. Overall, the results in Figure 10 demonstrate that the antenna temperature differences after anomaly correction throughout a year are usually smaller than $0.5 \mathrm{~K}$. Therefore, a stable performance of the SSMIS CTDR radiances at the LAS channels can be produced from the newly developed SSMIS anomaly correction algorithm.

Furthermore, for monitoring the onorbit instrument performance and for achieving the calibration consistency and traceability, the intersatellite comparison among various satellite-borne radiometers is very important. Since SSMIS and AMSU-A have several channels in common, the measurements at 53.6, 54.4, 55.5, and $57.3 \mathrm{GHz}$ from both instruments can be compared directly for calibration consistency check-up. To match the data together, we developed the simultaneous conical overpass (SCO) which is a slight revision of the simultaneous Nadir overpass (SNO) algorithm $[18,22]$. When two satellites at different altitudes cross each other at the same earth location, an SCO match pair is generated. The distance window from two sensor observations is set to be equal to an earth ground distance of $50 \mathrm{~km}$; the time window is set to 60 seconds; the viewing angle window is about $2^{\circ}$.

There are a total of 347 SCO events found for F-16 SSMIS and NOAA-16 AMSU from January 2005 to December 2006, which are located usually at high latitudes. The brightness temperatures of these SCO-observations at 53.6, 54.4, 55.5, and $57.3 \mathrm{GHz}$ are displayed in Figures 11(a)11(d), respectively. For convenience of comparison, the SCO observations of SSMIS brightness temperatures (SDR data) before and after our new calibration are plotted against AMSU measurements. The mean brightness temperature difference and the standard deviation of the brightness temperature differences are shown in the figures. It is recognized that the original SSMIS brightness temperatures are larger than these from NOAA-16 AMSU by a positive value from 1 and $2 \mathrm{~K}$ depending upon channel, which is consistent with the previous analyses. The mean differences in brightness temperatures between calibrated SSMIS and 


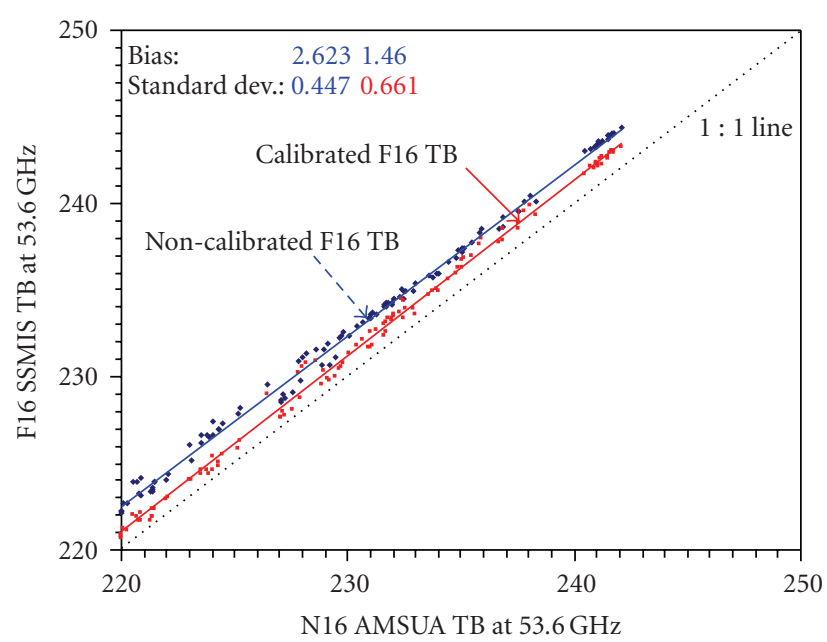

(a)

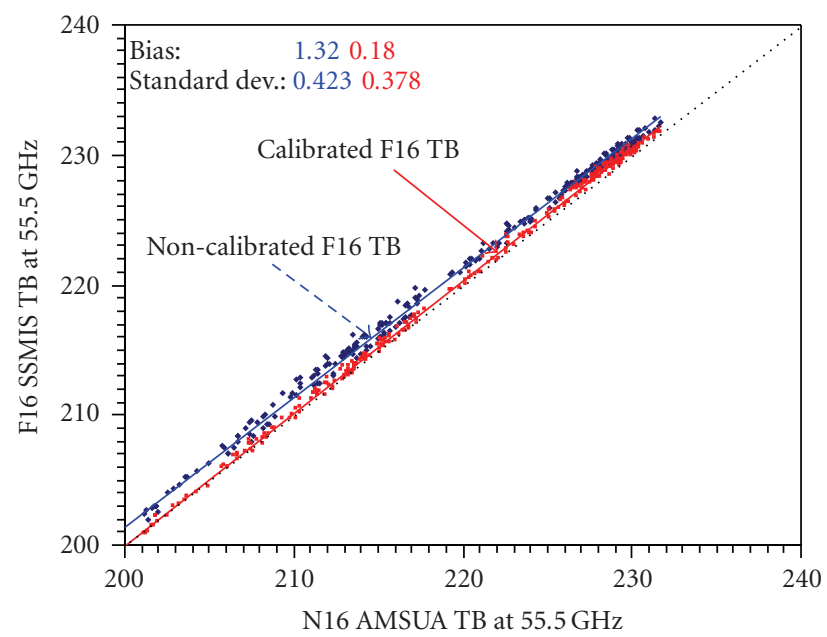

(c)

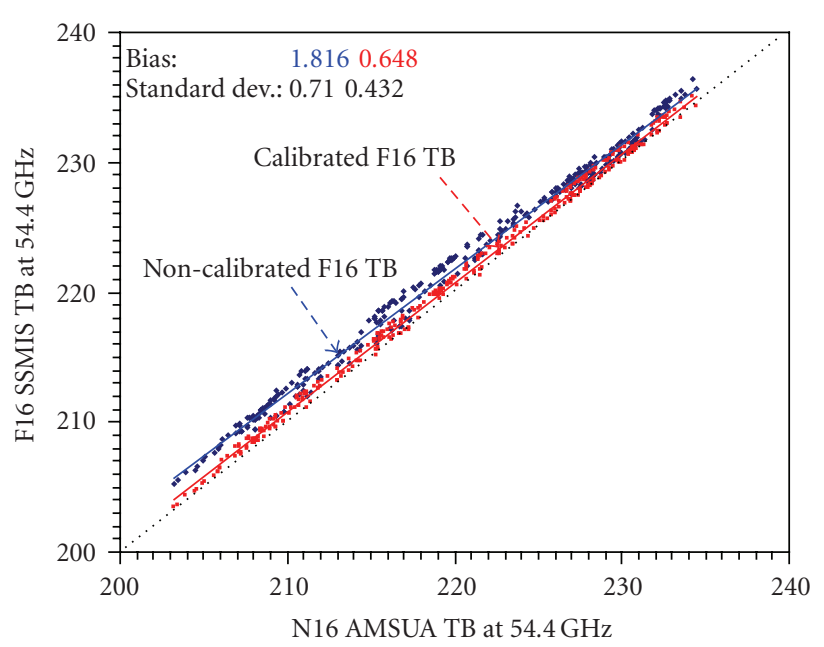

(b)

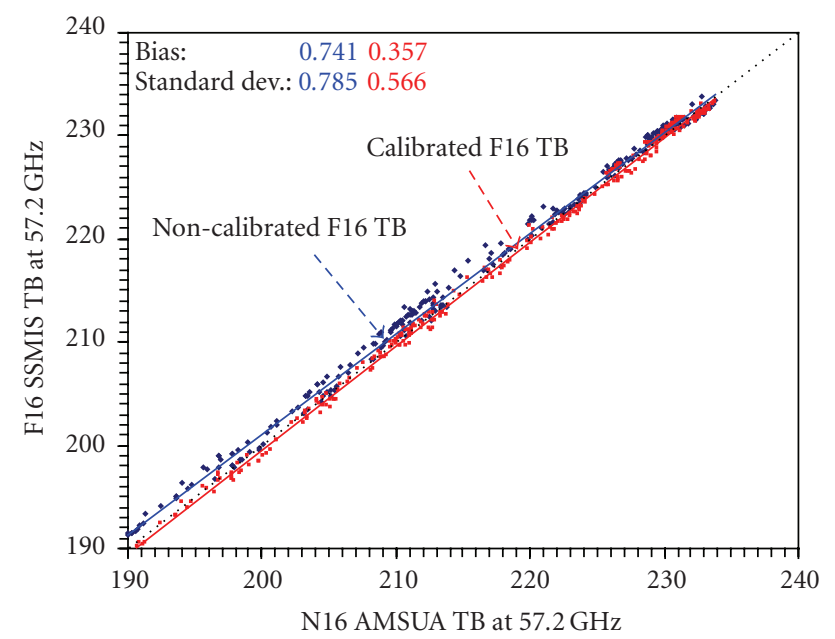

(d)

FIGURE 11: Intersatellite comparisons of brightness temperatures (TB) between F16 SSMIS TDR (CTDR) and NOAA-16 measurements at (a) $53.6 \mathrm{GHz}$, (b) $54.4 \mathrm{GHz}$, (c) $55.5 \mathrm{GHz}$, and (d) $57.3 \mathrm{GHz}$. In the figures, red is for newly calibrated while blue for original TDR results.

NOAA-16 AMSU measurements are usually around $0.5 \mathrm{~K}$ at $54.4,55.5$, and $57.3 \mathrm{GHz}$. At $53.6 \mathrm{GHz}$, the mean differences for both TDR and CTDR SSMIS data are relatively large, which might imply a large systematic bias in F16 SSMIS data at this frequency.

As shown in Figures 11(a)-11(d), the standard deviation of CTDR at each of the four channels is between 0.3 and 0.5 , which is usually smaller than that of TDR data except for $53.6 \mathrm{GHz}$. This implies that the aforementioned SSMIS recalibration algorithm corrects the major effects of the antenna emission and the solar intrusion on the warm counts, but at the same time, it adds some noises into the CTDR data at $53.6 \mathrm{GHz}$. The noise sources associated are investigated by two experiments, turning on/off emission and warm count anomaly correction prior SCO collocation. Table 3 displays the mean brightness temperature biases and standard deviation between AMSU-A and SSMIS brightness temperatures. Here, three F16 datasets are used including original TDR data, TDR data with only emission anomaly correction, and the TDR data including both emission and warm count anomaly corrections. It is found that the emission correction contributes some extra noises to the data (i.e., a larger standard deviation compared to the TDR data) at 53.6 and $55.5 \mathrm{GHz}$. The warm count anomaly correction reduces the standard deviation. An increase in the standard deviation from the antenna emission correction may be due to inaccurate reflector temperatures (see Section 4.3 for details).

\subsection{Impacts of Anomaly-Reduced SSMIS Measurements on} NCEP NWP Model. Direct benefits from improved cal/val algorithms will be more satellite data used in NWP models, which is directly related to quality of satellite data. So, it is important to demonstrate if the anomaly-corrected SSMIS data at the LAS channels is of good quality for operational applications in weather and climate models. The anomalies in F16 SSMIS radiances at the LAS channels 
TABLE 3: Biases and standard deviation (STD) characteristics of brightness temperature differences between F16 SSMIS and NOAA 16 AMSU from SCO measurements at four frequencies.

\begin{tabular}{|c|c|c|c|c|c|}
\hline TDR type & BIAS/STD & $53.6 \mathrm{GHz}$ & $54.4 \mathrm{GHz}$ & $55.5 \mathrm{GHz}$ & $57.2 \mathrm{GHz}$ \\
\hline \multirow{2}{*}{ No recalibration } & BIAS & 2.623 & 1.816 & 1.320 & 0.741 \\
\hline & STD & 0.447 & 0.710 & 0.423 & 0.785 \\
\hline \multirow{2}{*}{ Emission only correction } & BIAS & 1.433 & 0.458 & 0.117 & 0.622 \\
\hline & STD & 0.722 & 0.471 & 0.479 & 0.551 \\
\hline \multirow{2}{*}{ Emission plus warm count anomaly corrections } & BIAS & 1.460 & 0.648 & 0.180 & 0.357 \\
\hline & STD & 0.661 & 0.432 & 0.378 & 0.566 \\
\hline
\end{tabular}

can be mostly corrected using the algorithm as shown in Section 3. However, there remain certain scan-dependent biases in F16 SSMIS measurements which are irrelevant to calibration anomaly [10, 23]. Figures $12(\mathrm{a})-12(\mathrm{~d})$ display mean biases of antenna temperatures against scan beam position at four LAS channels, which is a difference between antenna temperature at a given beam position and that at a central beam position. The results in Figure 12 represent an average based on one year of data in ascending node over cloud-free oceans. An obvious decrease of around a couple of Kevin depending on channel is detected at each channel. Since the scan-dependent bias feature is relatively stable, this scan bias can be corrected in terms of scan position from the SSMIS measurements. NWP model is based on an assumption that the used data is no bias. A bias correction including SSMIS scan bias is thus needed as the SSMIS data is applied to NWP (the description of the bias correction is omitted here). Figure 13(a) displays the utilization rate of the SSMIS data with a bias correction at channels 1-4 for three orbits of observations which are assimilated into the NCEP global forecast model after a set of quality controls (QCs) (seeing explanations in the figure for details). For comparison, the data recalibrated using UK SSMIS recalibration algorithm (UKMO SSMIS CTDR data) [21] is assimilated into the NCEP global forecast model and the data usage is also shown in the figure. It is found that NESDIS TDR anomaly correction algorithms result in more SSMIS data assimilated into NWP models than the UK SSMIS recalibration algorithm. For instance, about 40 to $70 \%$ of NESDIS SSMIS CTDR data at channels 2 and 4 can be assimilated into the NCEP global forecast system, comparing to only 15 and 60\% if UKMO SSMIS CTDR data are used.

With those SSMIS CTDR data, the positive impact is also observed in predicting the NWP analysis fields such as geopotential height. Figure 13(b) is the distribution of difference of root mean square error (RMSE) of two geopotential heights versus latitude, where two geopotential heights are predicted by using "Test" and "Cntl" assimilation experiments correspondingly. In "Cntl" assimilation experiment, only operational global dataset including AMSU data is used in NCEP gridpoint statistical interpolation (GSI) global data assimilation system, whereas in "Test" experiment, NESDIS SSMIS CTDR data is also added into the GSI system in addition to the operational and AMSU datasets. Results in the figure do show that a negative difference in the RMSE of the geopotential height at various latitudes in the stratosphere, which implies that the use of NESDIS SSMIS CTDR data improves the accuracy of the geopotential height. In the lower atmosphere, the obvious improvement is also detected as the NESDIS SSMIS CTDR data is used in the GSI system. These results further demonstrated the good performance of the NESDIS SSMIS CTDR data at the LAS channels. For comparison, a similar assimilation experiment to the NESDIS SSMIS CTDR data experiment except for UKMO SSMIS CTDR data is carried out. The corresponding RMSE vertical distribution in comparison with the control experiment is also displayed in Figure 13(b).

4.3. Error Discussion. The above anomaly-corrected SSMIS antenna temperatures at the LAS channels show a good agreement with the corresponding RTM simulations and AMSU-A observations, and produce some positive impact on numerical weather prediction analysis fields. However, since some approximations are assumed in the derived anomaly correction algorithm, it is still important to investigate any possible errors in the algorithm.

One error source may stem from the used main reflector emissivity. Since the onboard F16 SSMIS main reflector emissivity at the LAS channels is not available, the SSMIS main reflector emissivity in this study relies on the NRL multilayer antenna model simulations. Some studies have shown that the NRL multilayer antenna model simulations are imperfect [24]. According to definition of antenna emission (see Section 2.3), for an emissivity error of $\delta \varepsilon_{R}$, the error of antenna emission equals to $\left(T_{R}-T_{A}\right) \delta \varepsilon_{R}$ when an accurate reflector temperature is used. Fortunately, in this study, the training set of reflector temperatures at several LAS channels is determined using (9) for known reflector emissivity and antenna emission (see Section 3.2). Thus, (9) is rewritten to be

$$
\varepsilon_{R}\left(T_{R}-T_{A}\right)=T_{\mathrm{AE}},
$$

where $T_{\mathrm{AE}}$ is antenna emission defined in Section 2.3 and also equals to $T_{A}^{\prime}-T_{A}$. Note that the channel frequency and polarization of each variable are omitted here. For the antenna emissions at channels $4-7$, that is, $T_{A}^{\prime}-T_{A}$, they are obtained by differences between SSMIS measured and RTM simulated brightness temperatures. This implies that the error in used antenna emission at each of channels 4-7 is only related to RTM simulation accuracy, but it is irrelevant to the errors in $\varepsilon_{R}$ and $T_{R}$. At channels 1-3, 


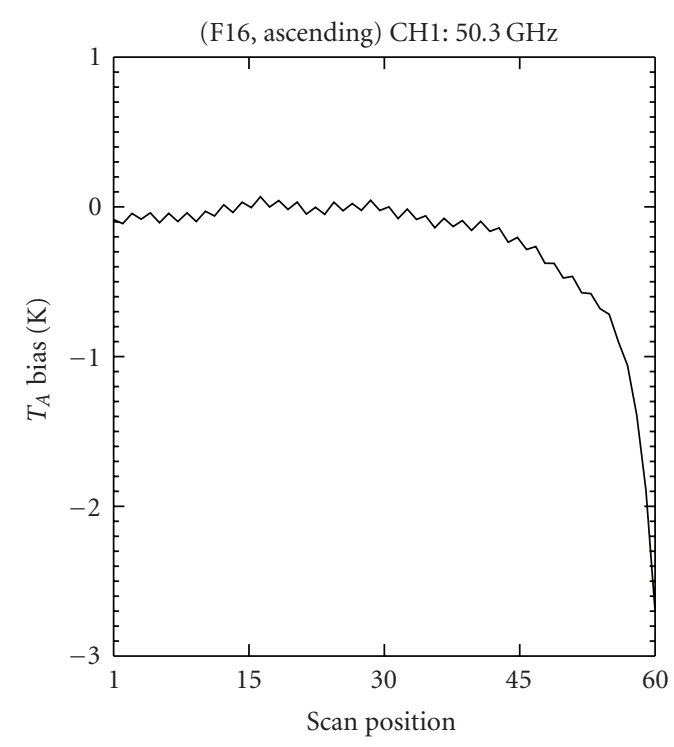

(a)

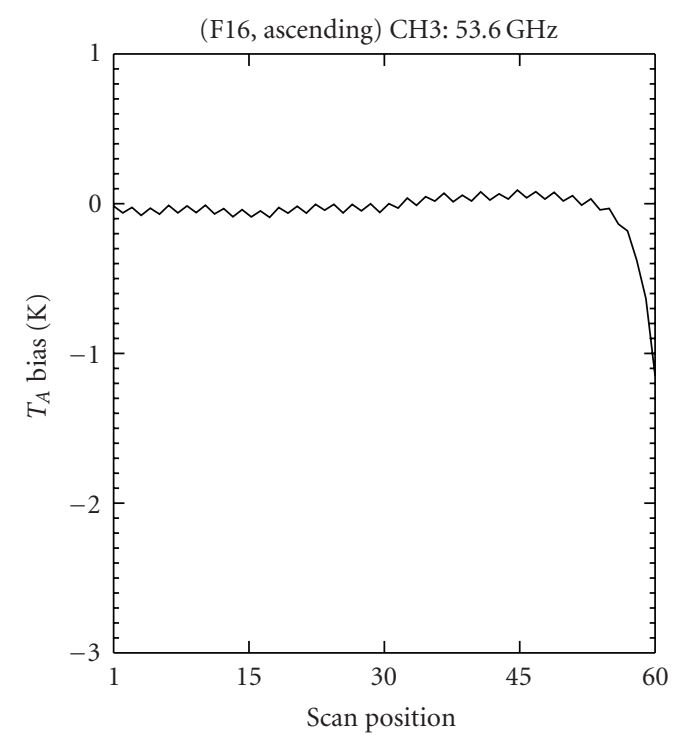

(c)

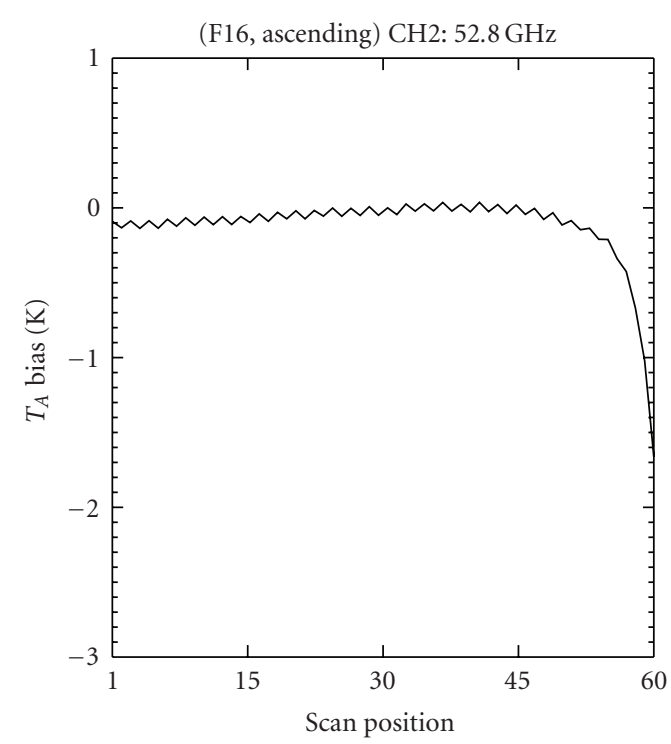

(b)

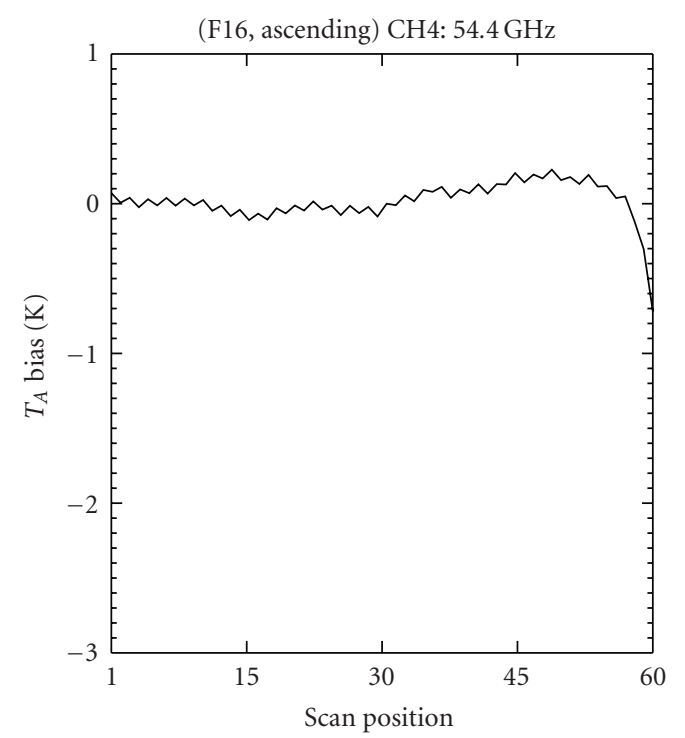

(d)

Figure 12: SSMIS antenna temperature $\left(T_{A}\right)$ scan-dependent bias in ascending node at several LAS channels [23]. (a) 50.3 GHz. (b) 52.8 GHz. (c) $53.6 \mathrm{GHz}$. (d) $54.4 \mathrm{GHz}$.

the reflector temperatures are assumed to be the same as that at channel 4. A certain error can be caused by this inaccurate assumption or the difference between the reflector temperature at channels 1-3 and that at channel 4. According to our analysis over oceans, this difference is relatively small and so-caused effect on antenna temperature is also small (an estimate of this effect is given below). Thus, the accuracies of the antenna emissions and anomaly-corrected antenna temperatures at the LAS channels are less affected by the error in the reflector emissivity in this study.

However, the error in $\varepsilon_{R}$ can be transferred to $T_{R}$ such that the reflector temperature derived using (9) may be not real reflector temperature if the NRL emissivity simulations deviate obviously from real F16 reflector properties. This can be seen by applying an operator of derivative to (11), that is,

$$
\delta T_{R}=-\frac{\left(T_{R}-T_{A}\right) \delta \varepsilon_{R}}{\varepsilon_{R}+\delta \varepsilon_{R}} .
$$

Thus, there would remain some uncertainty in derived reflector temperatures at LAS channels. In addition, potential error in reflector temperature due to emissivity error implies that the antenna emission correction algorithm shown in Section 3.2 is inapplicable for window channels because an accurate reflector emissivity is always needed for accurate antenna emission simulations and corrections at window channels. For example, at 19.35, 23.8, 31.4, $91.655 \mathrm{GHz}$, the accurate RTM simulations over global areas are at least 


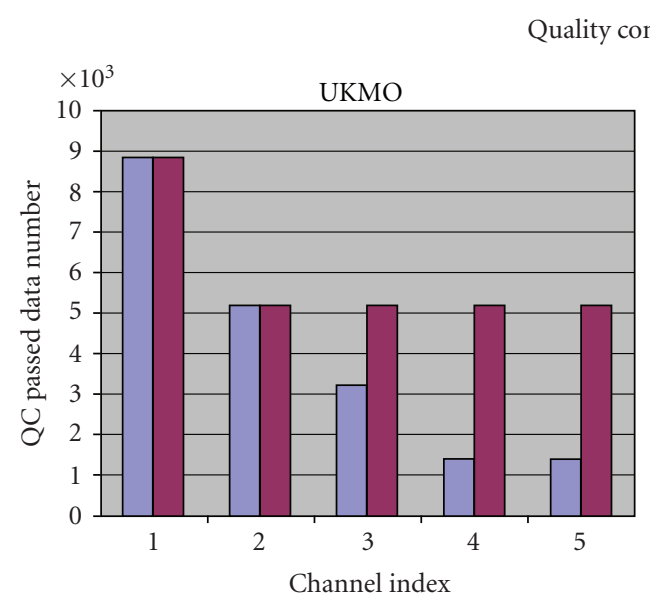

SSMIS CH2: $15 \%$ data were used after QC SSMIS CH4: 60\% data were used after QC

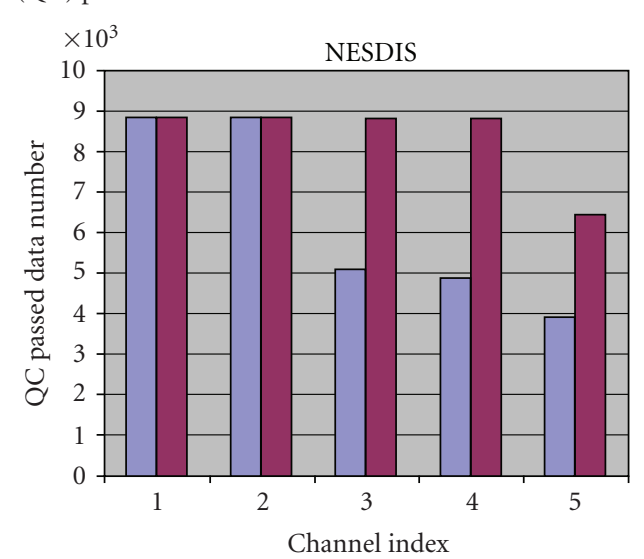

SSMIS CH2: $44 \%$ data were used after QC SSMIS CH4: 72\% data were used after QC
QC1: after thinning

QC2: solar contamination and rain flag QC3: land ice QC QC4: cloud QC QC5: gloss error check

(a)
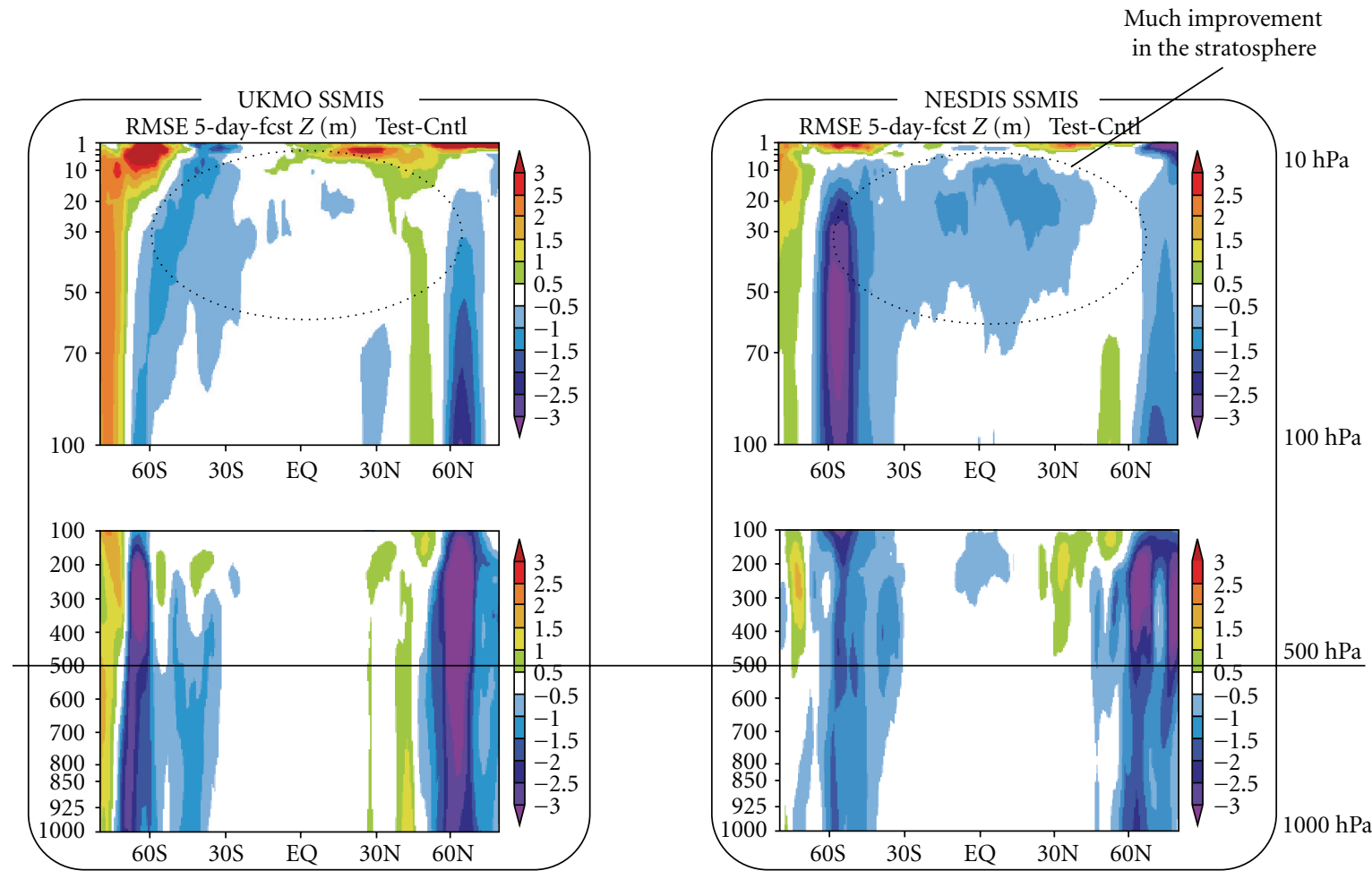

- Blue color means improvement.

(b)

FIGURE 13: (a) Number of the SSMIS data from channel 1 to channel 4 for three orbits of observations used in the NCEP global forecast model, on August 9, 2006. (b) Vertical distribution of root mean square error (RMSE) difference in the predicted geopotential height along latitude between the five days of assimilation experiments of "Cntl" and "Test" by using the NCEP global forecast model (Courtesy to Masahiro Kazumori).

difficult if not impossible due to our limited knowledge of emissivity simulation over land. These channels also use different feedhorns from LAS channels, so the approximation of the reflector temperature at channel 4 to these window channels would be invalid. It becomes very important to have accurate reflector emissivity and temperature for reliable assessment of antenna emission there using definition of antenna emission.

Another error source may result from the reflector temperature error caused by other three possible error sources: (1) the assumption that $T_{R, \mathrm{CH} 1-3} \approx T_{R, \mathrm{CH} 4} ;$ (2) the assumption that the RTM simulations are equal to realistic 
anomaly-free radiances; (3) the reflector temperature bias due to the inaccurate fitting (see Figure 7). This error can be expressed to be

$$
\delta T_{A}=\varepsilon_{R} \delta T_{R}^{\prime} .
$$

Note that this reflector temperature error $\left(\delta T_{R}^{\prime}\right)$ is different from that defined by (12) because this error is not correlated to the emissivity error. The reflector temperature error due to RTM simulation error is usually smaller than $10 \mathrm{~K}$ because RTM simulation error is about $0.2 \mathrm{~K}$, while the reflector temperature error due to other factors are also relatively small. As shown in Sections 4.1 and 4.2, the total residual error in anomaly-corrected antenna temperatures is about $0.5 \mathrm{~K}$. Therefore, the SSMIS CTDR data is of sufficient accuracy to be used operationally for input to numerical weather prediction and climate models.

\section{Summary and Conclusions}

F16 SSMIS radiances at LAS channels show certain anomalies approximately between $-1 \mathrm{~K}$ and $2 \mathrm{~K}$ due to both antenna emission and solar heating of warm load tines. The antenna emission occurs globally and results in a strong geographical dependence with obvious peaks frequently near the mid- and high-latitudes, which can result in either positive or negative error in observed antenna temperatures depending on difference between reflector temperature and earth-viewing antenna temperature. The warm load count anomaly occurs over several latitudinal zones whose coverage is around 30 $40 \%$ of each orbit of observations, which always results in a negative error in observed antenna temperatures.

An algorithm for SSMIS antenna temperature anomaly correction has been developed using the F16 SSMIS TDR data based on FFT analysis and calibration equation associated with reflector temperature. With this SSMIS anomaly correction algorithm based on the SSMIS TDR data, most of the effects from the warm count and antenna anomalies can be removed from the SSMIS observations at the LAS channels with a residual error smaller than $0.5 \mathrm{~K}$. The produced SSMIS CTDR data is much encouraging and is also of sufficient accuracy to be used operationally for input to numerical weather prediction and climate models. A direct benefit from this SSMIS CTDR data is more SSMIS data being used in NWP models, resulting in improved NWP analysis fields. The F16 CTDR data is archived in NOAA STAR and available under request through fuzhong.weng@noaa.gov and banghua.yan@noaa.gov.

However, there remain some weaknesses in this study. For example, the NRL-modeled emissivity used in this study has certain errors [24]. This error would not obviously affect the accuracy of the SSMIS antenna temperatures with anomaly correction at several LAS channels. However, this error can result in some errors in the derived reflector temperatures and can affect an accurate assessment of antenna emission at window channels. Therefore, more studies are needed in our future research activities about the best estimates of F16 main reflector emissivity and temperatures at various channels.

\section{Acknowledgments}

Authors would like to thank the editor Dr. Zhanqing Li and several anonymous reviewers for their very useful suggestions to make the paper better. Thanks also go to Mr. Steve Swadley, Dr. William Bell, and Mrs. Ninghai Sun for their helps. A special thank goes to Masahiro Kazumori for testing NESDIS SSMIS CTDR data in NCEP NWP model. The views expressed in this publication are those of the authors and do not necessarily represent those of NOAA.

\section{References}

[1] G. Poe, K. Germain, J. Bobak, et al., "DMSP Calibration/Validation plan for the Special Sensor Microwave Imager Sounder (SSMIS)," Tech. Rep., Naval Research Laboratory, Washington, DC, USA, 2001.

[2] N. Grumman, "Algorithm and data user manual (ADUM) for the Special Sensor Microwave Imager/Sounder (SSMIS)," Tech. Rep. 12621, Northrop Grumman Corporation, Space Systems Division, Azusa, Calif, USA, 2002.

[3] S. Swadley, G. Poe, A. Uliana, and D. Kunkee, "SSMIS Cal/Val calibration anomaly analysis," in NOAA-JCSDA Seminar, Washington, DC, USA, September 2005.

[4] S. Swadley, G. Poe, A. Uliana, and D. Kunkee, "SSMIS radiance correction anomalies," in Proceedings of the 1st SSMIS Working Group Meeting, Monterey, Calif, USA, 2005.

[5] S. Swadley, G. Poe, A. Uliana, and D. Kunkee, "SSMIS regression based anomaly corrections," in Proceedings of the 1st SSMIS Working Group Meeting, Monterey, Calif, USA, 2005.

[6] W. Bell, S. English, and S. Swadley, "SSMIS calibration issues," in Proceedings of the 1st SSMIS Working Group Meeting, Monterey, Calif, USA, 2005.

[7] B. Yan, F. Weng, and T. Mo, "Calibration of DMSP F-16 Special Sensor Microwave Imager and Sounder," in Proceedings of the 14th Conference on Satellite Meteorology and Oceanography, Atlanta, Ga, USA, January-February 2006.

[8] B. Yan, F. Weng, N. Sun, and H. Xu, "Assessments of F16 Special Sensor Microwave Imager and Sounder Data for NOAA Operational Applications," in Proceedings of the 9th Specialist Meeting on Microwave Radiometry and Remote Sensing Applications (MicroRad '06), pp. 18-23, San Juan, Puerto Rico, February-March 2006.

[9] W. Bell, B. Candy, N. Atkinson, et al., "The assimilation of SSMIS radiances in numerical weather prediction models," IEEE Transactions on Geoscience and Remote Sensing, vol. 46, no. 4, pp. 884-900, 2008.

[10] D. B. Kunkee, G. A. Poe, D. J. Boucher, et al., "Design and evaluation of the first Special Sensor Microwave Imager/Sounder," IEEE Transactions on Geoscience and Remote Sensing, vol. 46, no. 4, pp. 863-883, 2008.

[11] F. Weng, B. Yan, and N. Sun, "Correction of SSMIS radiance anomalies," in Proceedings of the 1st SSMIS Working Group Meeting, Monterey, Calif, USA, 2005.

[12] F. Weng, Y. Han, P. van Delst, Q. Liu, and B. Yan, "JCSDA Community radiative transfer model (CRTM)," in Proceedings of 14th International ATOVS Study Conference, Beijing, China, May 2005.

[13] P. W. Rosenkranz, "Water vapor microwave continuum absorption: a comparison of measurements and models," Radio Science, vol. 33, no. 4, pp. 919-928, 1998. 
[14] S. J. English and T. J. Hewison, "A fast generic millimetrewave emissivity model," in Microwave Remote Sensing of the Atmosphere and Environment, vol. 3503 of Proceedings of SPIE, pp. 288-300, Beijing, China, September 1998.

[15] J. Hollinger, R. Lo, G. Poe, R. Savage, and J. Peirce, "Special sensor microwave/imager users' guide," Tech. Rep., Naval Research Laboratory, Washington, DC, USA, 1987.

[16] N. C. Grody, K. Y. Vinnikov, M. D. Goldberg, J. T. Sullivan, and J. D. Tarpley, "Calibration of multisatellite observations for climatic studies: microwave Sounding Unit (MSU)," Journal of Geophysical Research, vol. 109, no. D24, 2004.

[17] C.-Z. Zou, M. D. Goldberg, Z. Cheng, et al., "Recalibration of microwave sounding unit for climate studies using simultaneous nadir overpasses," Journal of Geophysical Research, vol. 111, no. 19, Article ID D19114, 2006.

[18] B. Yan and F. Weng, "Intercalibration between Special Sensor Microwave Imager/Sounder and Special Sensor Microwave Imager," IEEE Transactions on Geoscience and Remote Sensing, vol. 46, no. 4, pp. 984-995, 2008.

[19] S. Swadley, G. Poe, N. Baker, et al., "Calibration anomalies and radiance assimilation correction strategies for the Defense Meteorological Satellite Program (DMSP) Special Sensor Microwave Imager Sounder (SSMIS)," in Proceedings of the 15th International TOVS Studies Conference, Maratea, Italy, October 2006.

[20] E. M. Twarog, W. E. Purdy, P. W. Gaiser, K. H. Cheung, and B. E. Kelm, "WindSat on-orbit warm load calibration," IEEE Transactions on Geoscience and Remote Sensing, vol. 44, no. 3, pp. 516-529, 2006.

[21] W. Bell, "A preprocessor for SSMIS radiances scientific description," MET Office, UK, 2006, http://www.metoffice .com/research/interproj/nwpsaf/ssmis_pp/H.

[22] C. Cao, M. Goldberg, F. Weng, C. Zou, and P. Ciren, "Simultaneous Nadir Overpasses for NOAA-6 to NOAA-17 satellites from 1980 to 2003 for the intersatellite calibration of radiometers," Tech. Rep. NESDIS 118, NOAA, Washington, DC, USA, 2005.

[23] B. Yan and F. Weng, "Recalibration of DMPS SSM/I for weather and climate applications," in Proceedings of the 15th International TOVS Studies Conference, Maratea, Italy, October 2006.

[24] D. Boucher, G. Poe, and Coauthors, "Defense meteorological satellite program Special Sensor Microwave Imager Sounder (F-16) Calibration/Validation," Final Report, November 2005. 

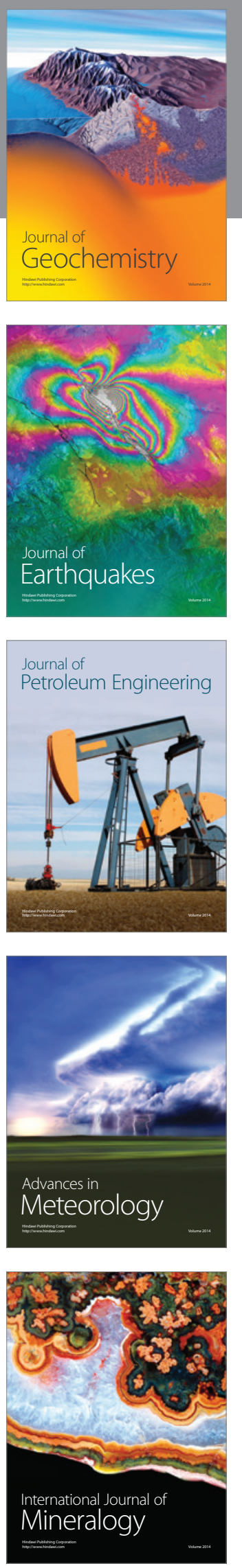
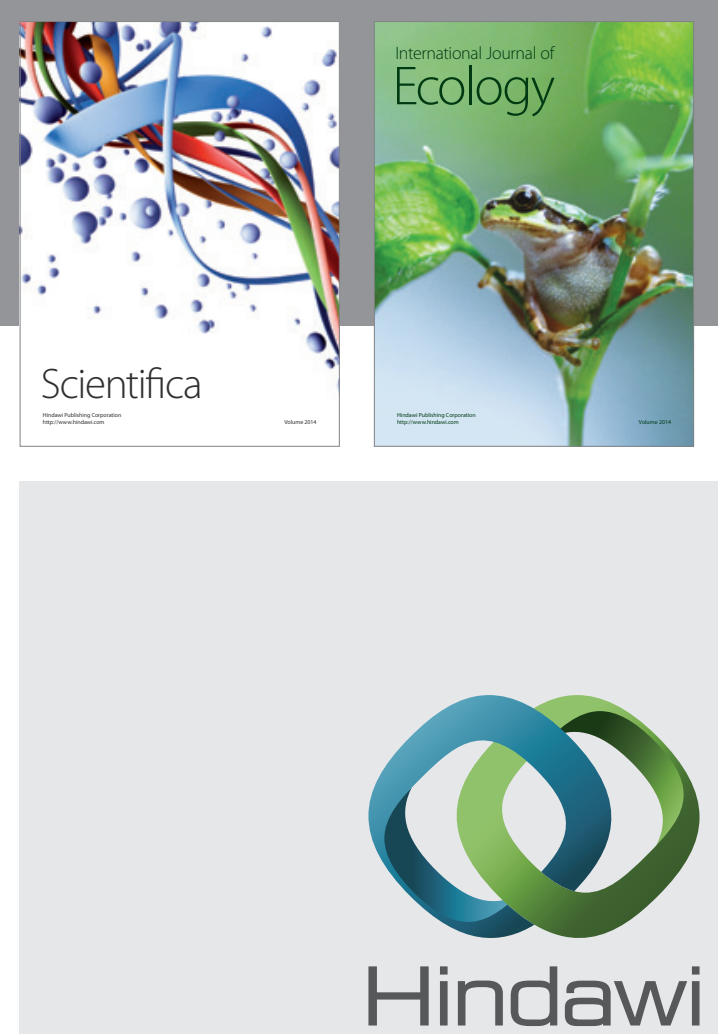

Submit your manuscripts at http://www.hindawi.com
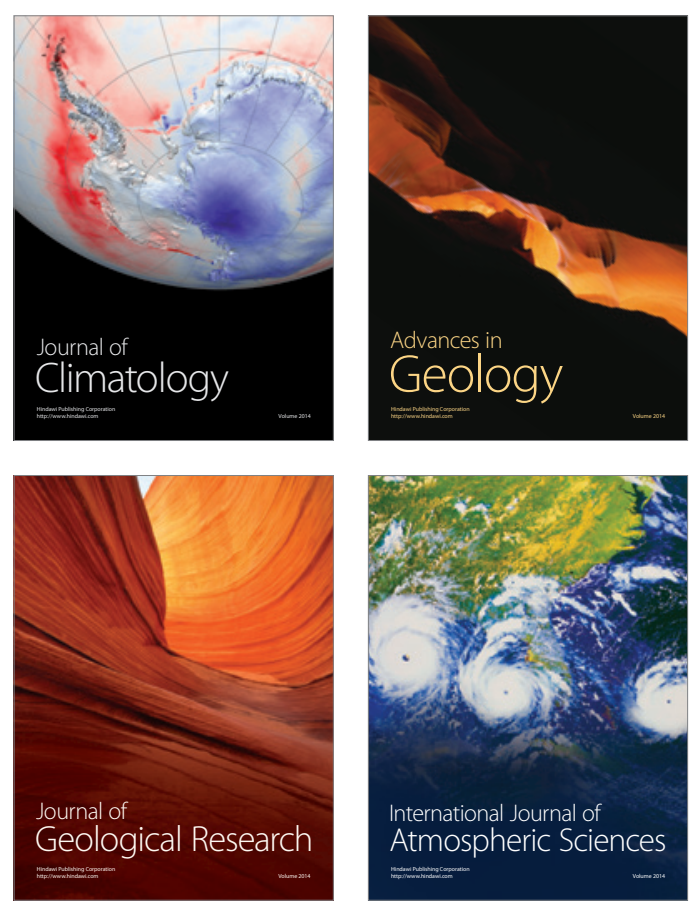
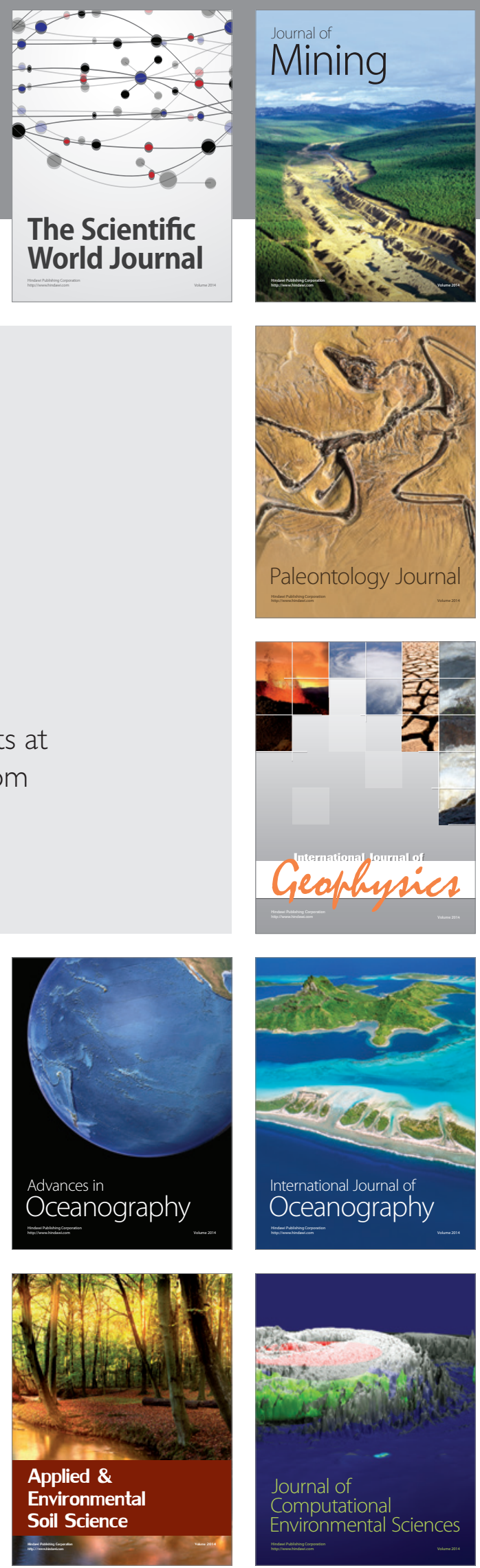